\title{
Acquisition of the contractile phenotype by murine arterial smooth muscle cells depends on the Mir143/145 gene cluster
}

\author{
Thomas Boettger, ${ }^{1}$ Nadine Beetz, ${ }^{2}$ Sawa Kostin, ${ }^{1}$ Johanna Schneider, ${ }^{2}$ \\ Marcus Krüger, ${ }^{1}$ Lutz Hein, ${ }^{2}$ and Thomas Braun' \\ ${ }^{1}$ Max-Planck-Institut für Herz- und Lungenforschung, Bad Nauheim, Germany. ${ }^{2}$ Albert-Ludwigs-Universität Freiburg, \\ Institut für Experimentelle und Klinische Pharmakologie und Toxikologie, Freiburg, Germany.
}

\begin{abstract}
VSMCs respond to changes in the local environment by adjusting their phenotype from contractile to synthetic, a phenomenon known as phenotypic modulation or switching. Failure of VSMCs to acquire and maintain the contractile phenotype plays a key role in a number of major human diseases, including arteriosclerosis. Although several regulatory circuits that control differentiation of SMCs have been identified, the decisive mechanisms that govern phenotypic modulation remain unknown. Here, we demonstrate that the mouse miR-143/145 cluster, expression of which is confined to SMCs during development, is required for VSMC acquisition of the contractile phenotype. VSMCs from miR-143/145-deficient mice were locked in the synthetic state, which incapacitated their contractile abilities and favored neointimal lesion development. Unbiased high-throughput, quantitative, mass spectrometry-based proteomics using reference mice labeled with stable isotopes allowed identification of miR-143/145 targets; these included angiotensin-converting enzyme (ACE), which might affect both the synthetic phenotype and contractile functions of VSMCs. Pharmacological inhibition of either $\mathrm{ACE}$ or the $\mathrm{AT}_{1}$ receptor partially reversed vascular dysfunction and normalized gene expression in miR-143/145-deficient mice. We conclude that manipulation of miR-143/145 expression may offer a new approach for influencing vascular repair and attenuating arteriosclerotic pathogenesis.
\end{abstract}

\section{Introduction}

VSMCs are highly specialized cells that regulate the lumenal diameter of small arteries/arterioles called resistance vessels, thereby contributing significantly to the regulation of blood pressure. The tone of VSMCs is controlled by angiotensin II and adrenergic stimulation, although numerous other signaling molecules affect the contractile status of VSMCs (1). VSMCs proliferate rarely under normal physiological conditions in adult tissues but will undergo major phenotypic changes from the contractile to the synthetic phenotype in response to environmental cues such as hypertension, vascular injury, and arteriosclerosis (2). Early atherosclerotic lesions are often characterized by focal accumulation of VSMCs within the intima. The exact function of VSMCs in atherosclerosis, however, is still a matter of debate (3). VSMCs might contribute to the development of atherosclerotic plaques by synthesizing proinflammatory mediators, vascular cell adhesion molecules, and matrix molecules required for the retention of lipoproteins (4). The ability for contraction, proliferation, and secretion depends on the differentiation status of VSMCs, which is affected by a large number of factors, including mechanical forces, contractile agonists such as angiotensin II, extracellular matrix components, neuronal factors, reactive oxygen species, endothelial-VSMC interactions, thrombin, PDGF, and TGF- $\beta 1$. Transcriptional responses to these factors are mostly mediated by serum response factor (SRF) and its cofactor myocardin, which play a key role in the regulation of VSMC markers, as well as by members of the Kruppel-like transcription factor fam-

Authorship note: Thomas Boettger and Nadine Beetz contributed equally to this work.

Conflict of interest: The authors have declared that no conflict of interest exists. Citation for this article: J. Clin. Invest. 119:2634-2647 (2009). doi:10.1172/JCI38864. ily of zinc finger proteins (reviewed in ref. 1). SRF-dependent gene transcription is controlled at various posttranscriptional levels, including - among others - differential splicing and phosphorylation- and RhoA/Rho kinase-dependent (ROK-dependent) SRF nuclear translocation and/or activation (reviewed in ref. 1).

In recent years, it has been unraveled that posttranscriptional regulation of gene expression critically depends on microRNAs (miRNAs), a class of approximately 22 nucleotide-noncoding RNAs, which repress protein expression of target mRNAs by mRNA degradation or translational repression (5). Using miRNA target prediction algorithms, hundreds of potential target mRNAs for a specific miRNA can be identified, and miRNA target binding sites have been predicted to be present in almost any transcript. Many miRNAs are expressed in a tissue-specific manner and play pivotal roles in the control of proliferation and differentiation of different cell types (6-9). To understand the role of miRNAs in the control of VSMC differentiation and function, we screened for the presence of miRNAs in organs with a high content of SMCs using miRNA microarray hybridization. We found that the expression of the miR-143/145 cluster becomes confined to SMCs during embryonic development. The miR-143/145 cluster is instrumental to the acquisition and/or maintenance of the contractile phenotype of VSMCs and controls the concentration of proteins that regulate contractility of the VSMCs. Loss of the miRNA cluster eventually results in deregulated blood pressure and formation of neointimal lesions in normolipidemic mice.

\section{Results}

Expression of miR-143/145 and generation of Mir143/145 mutant mice. To understand the role of miRNAs in the development and regulation of the cardiovascular system, we performed a series of miRNA 
A

\begin{tabular}{|c|c|c|c|c|c|c|c|c|}
\hline & KO aorta & aorta & bladder & lung & heart & muscle & brain & liver \\
\hline mmu-let-7a & 0.00 & 0.17 & 0.10 & -0.01 & -0.35 & 0.14 & -0.16 & 0.10 \\
\hline mmu-let-7b & 0.26 & 0.02 & 0.09 & -0.03 & -0.28 & -0.05 & -0.09 & 0.08 \\
\hline mmu-let-7c & 0.21 & 0.08 & 0.19 & 0.00 & -0.38 & -0.18 & 0.04 & 0.03 \\
\hline mmu-let-7d & 0.18 & 0.19 & -0.03 & -0.11 & -0.10 & 0.08 & -0.08 & -0.13 \\
\hline mmu-miR-143 & -2.29 & 3.08 & 4.14 & 0.64 & 0.58 & -2.82 & -2.06 & -1.29 \\
\hline mmu-miR-145 & -2.30 & 3.79 & 4.38 & 0.68 & 0.60 & -2.11 & -2.95 & -2.08 \\
\hline mmu-miR-1 & -1.88 & -1.22 & -0.22 & -2.21 & 4.09 & 5.88 & -2.22 & -2.22 \\
\hline mmu-miR-133a & -1.30 & -0.89 & -0.63 & -1.11 & 2.67 & 4.89 & -2.90 & -0.73 \\
\hline mmu-miR-9 & 0.22 & -0.51 & -0.67 & -1.97 & -0.77 & -0.11 & 3.46 & 0.36 \\
\hline mmu-miR-124 & -0.41 & -1.22 & -1.00 & -1.71 & 1.03 & -0.54 & 3.68 & 0.16 \\
\hline mmu-miR-122 & -0.89 & -0.11 & 0.10 & -1.28 & -0.98 & -1.56 & -0.87 & 5.59 \\
\hline
\end{tabular}

B

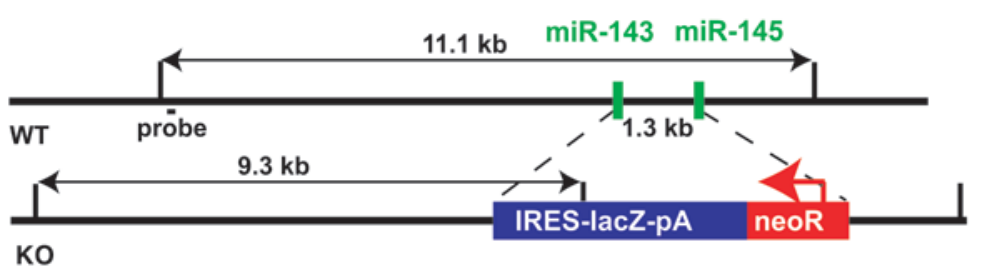

C

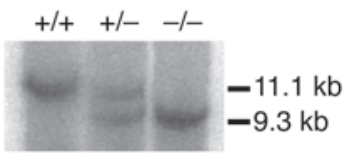

D

miRNA expression WT vs. KO aorta

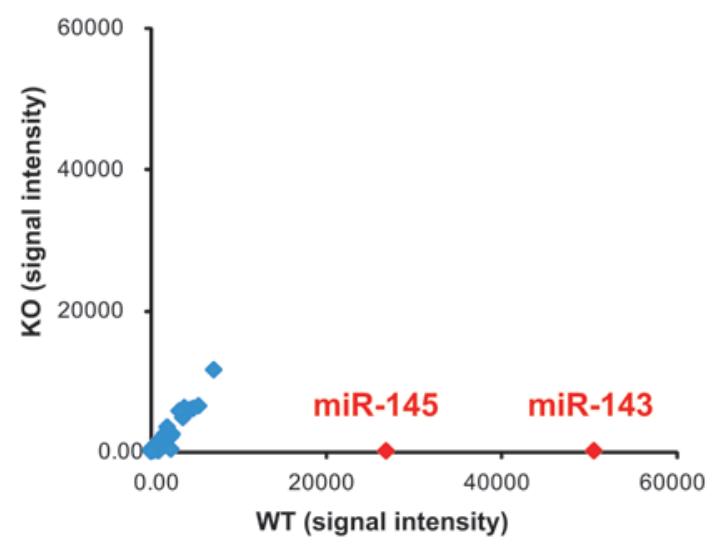

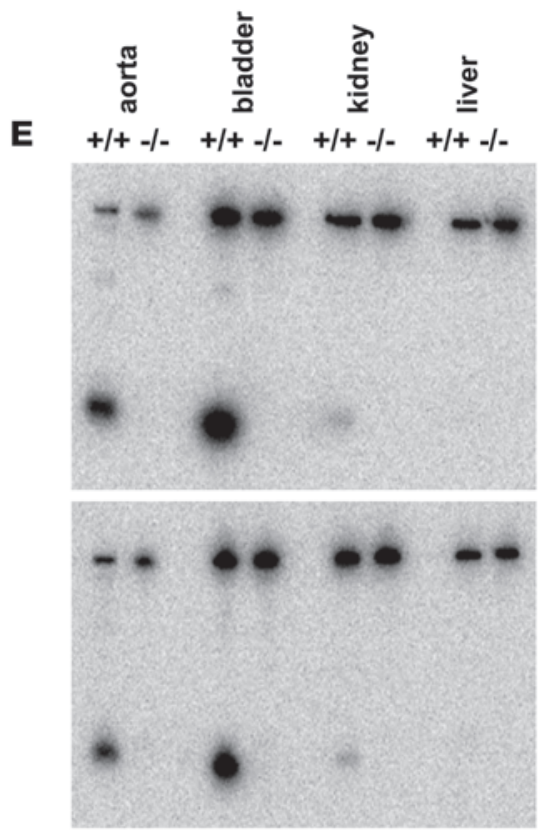

U6

miR-143

U6

miR-145

\section{Figure 1}

Microarray analysis of miR-143/145 expression and targeting strategy of the miR-143/145 cluster. (A) miRNA microarray analysis of different mouse tissues. Expression values are ratios of tissue-specific miRNA expression versus reference miRNA from an E15.5 mouse embryo. The miRNAs show a similar expression profile in different organs. The miR-1/133a cluster, miR-124/miR-9, and miR-122 are known markers of heart/muscle, brain, and liver, respectively. mmu, Mus musculus. (B) The miR-143 and miR-145 sequences are separated by a 1.3-kb fragment on mouse chromosome 18. Both miRNAs were deleted by insertion of an IRES-lacZ-Neo cassette. EcoRV restriction fragments used for genotyping are indicated. (C) Southern blot analysis of WT, heterozygous, and $\mathrm{KO}$ animals using the $5^{\prime}$ outside probe and EcoRV-digested genomic DNA. (D) Microarray analysis of miRNA expression of a WT versus KO aorta. Results of 2 microarray experiments with dye swapping for WT and KO mice are shown. The arrays were normalized to a ratio of medians of one for the let-7 signals, and the results of the 2 experiments were combined by calculating the mean of signals after reversion of all ratios $(1 / x)$ of the dye swap control experiment. miR-143 and miR-145 are shown in red; other miRNAs of the aorta are in blue. (E) Northern blot analysis of miRNA expression in WT and KO tissues. Blots were probed with radioactively labeled $U 6 / \mathrm{miR}-143$ and $\mathrm{U} 6 / \mathrm{miR}-145$, respectively.

microarray hybridization experiments using a number of different mouse tissues derived at various developmental stages. Our microarray analysis revealed that the expression of miR-143 and miR-145, which form a small cluster on mouse chromosome 18, strongly correlated with the number of SMCs in all organs studied
(Figure 1A). Detailed analysis of the function of miRNAs is often hampered by the lack of a robust expression map at the single-cell level. We therefore replaced the miR-143/145-coding genomic region with a lac $Z$ reporter by fusing the pre-miR-143 sequence to an IRESlac $Z$ sequence. This manipulation deleted the sequences coding for 

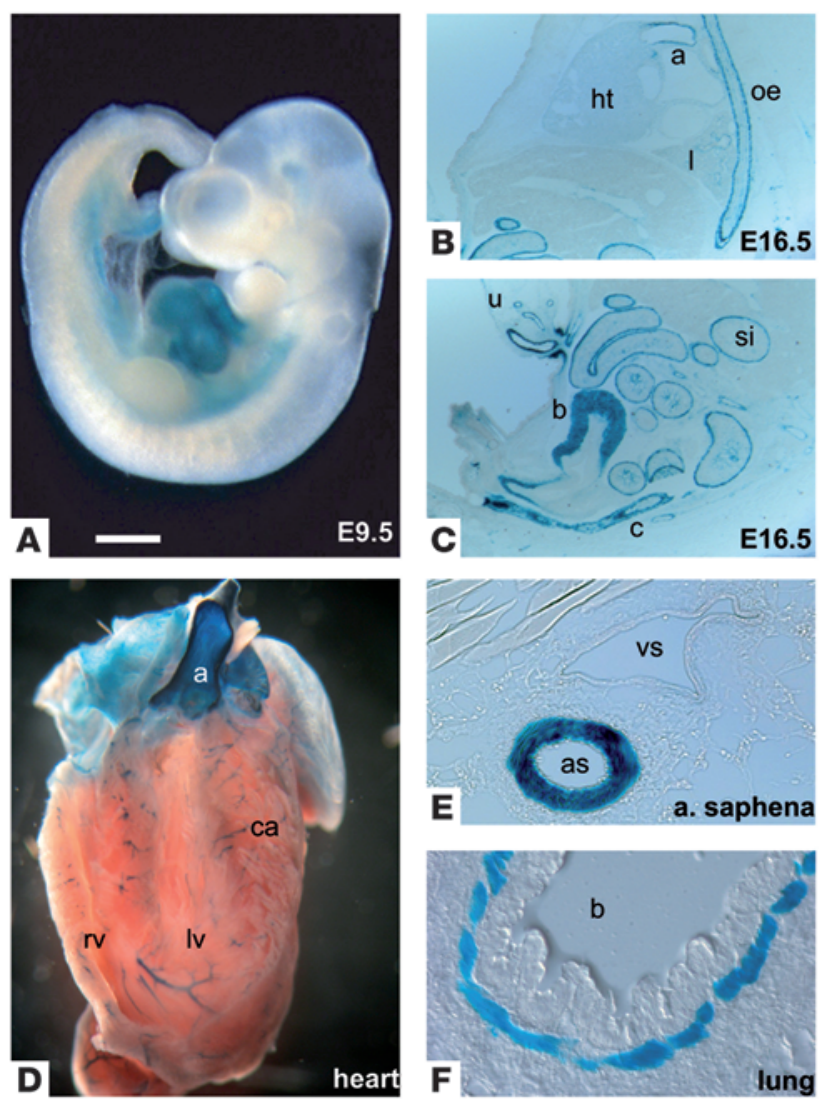

the mature miR-143 and miR-145 sequences and a 1.3-kb fragment located between the two genes (Figure 1, B and C) and allowed us to monitor transcription of the Mir143 gene. Examination of the activity of the lac $Z$ reporter in heterozygous mice revealed that the miR-143 locus was active during early stages of heart development from E8.5 onward (E9.5, Figure 2A) but disappeared from cardiomyocytes at later stages (E16.5, Figure 2B) and became exclusively confined to SMCs of the cardiovascular system, the gastrointestinal tract, the bladder, uterus, and lung (Figure 2, C-F). Interestingly, other smooth muscle genes such as $\alpha$-SMA and SM22 $\alpha$ follow a similar path and are also found in embryonic cardiomyocytes at early stages of heart development $(10,11)$. In the esophagus, an initial expression of the reporter was observed at $\mathrm{P} 0$ in the muscularis mucosae and the muscularis externa, which are composed of SMCs at this stage. In adult mice, expression of miR-143/145 was only found in the muscularis mucosae, since skeletal muscle cells supersede SMCs in the muscularis externa of the esophagus during postnatal development (Supplemental Figure 1; supplemental material available online with this article; doi:10.1172/JCI38864DS1) (12). The postnatal expression of the miR-143/145 cluster in VSMCs was relatively stable and did not respond to transstenotic pressure gradients induced by transverse aortic constriction ( $n=3$ aortic stenosis, $n=3$ control group; measured by microarray hybridization; data not shown).

Loss of miR-143/145 leads to a shift of contractile to synthetic VSMCs. To study the consequences of the loss of miR-143/145, we crossed heterozygous Mir143/145-KO animals. Homozygous mutants were viable and fertile and did not show gross macroscopic alterations. Microarray-based and Northern blot-based miRNA expression analysis of different $\mathrm{KO}$ tissues confirmed the loss of the expression

\section{Figure 2}

The miR-143/145 cluster is specifically expressed in the SMC lineage. (A-F) Specific expression of the lacZ reporter gene under control of the miR-143 promoter in SMCs. (A) miR-143/145 is initially expressed in the developing embryonic heart at E8.5 (not shown) to E9.5. (B and C) During fetal stages (E16.5), the expression of the miR-143 reporter gene becomes confined to SMCs of the aorta (a), smaller blood vessels, esophagus (oe), lung (I), small intestine (si), colon (c), bladder (b), and umbilical cord (u). (D-F) In adult animals, miR-143 expression is present in all SMCs throughout the body, including the aorta, the coronary arteries (ca) of the cross-sectioned heart, the saphenous artery (as), and the bronchi (b). Scale bar in A: $0.6 \mathrm{~mm}(\mathbf{A}) ; 0.9 \mathrm{~mm}$ (B and C); $1.2 \mathrm{~mm}(\mathbf{D}) ; 0.09 \mathrm{~mm}(\mathbf{E}) ; 0.06 \mathrm{~mm}(\mathbf{F})$.

of both miRNAs and demonstrated the specificity of the signals detected by microarray hybridization (Figure 1, D and E).

To obtain a more detailed view regarding the function of the Mir143/145 gene cluster, we performed a comprehensive analysis of the structure and function of VSMCs in vivo. Major structural changes in arterial VSMCs were noted in all arteries investigated (Figure 3). Electron microscopy analysis revealed a severe reduction in the number of contractile VSMCs and an increase in synthetic VSMCs in the aorta and the femoral artery of $\mathrm{KO}$ animals (Figure $3, \mathrm{~A}-\mathrm{C}$ and $\mathrm{H}-\mathrm{J}$ ), which went along with a reduced thickness of the smooth muscle layer of the femoral artery as demonstrated by electron microscopy (Figure 3, H-J) as well as by light microscopy (WT, $n=8: 32.9 \pm 1.4$ vs. KO, $n=7: 19.5 \pm 0.6 \mu \mathrm{m} ; P<0.0001)$. The phenotype switch of SMCs from contractile to synthetic was established by analysis of both smooth muscle shape and number of dense bodies per VSMC area (Supplemental Figure 3). Morphometric measurements indicated that the reduced size of the muscle layer was due to the smaller size of VSMCs (Supplemental Figures 3 and 4) and not to a reduced total number of VSMCs (Figure 3, H and I, and Supplemental Figures 3 and 4). The aorta showed a less severe phenotype compared with the femoral artery (data not shown). No differences in the number of proliferating or apoptotic SMCs was apparent in arteries of adult mutants (data not shown). The invariant total number combined with the reduction in the size of VSMCs reflected the shift between contractile and synthetic phenotypes and suggested that the loss of miR-143/145 affects not the differentiation of VSMCs per se but rather the ability to adjust the phenotype to the physiologically required contractile state.

The miR-143/145 cluster is required for normal contractility of arteries in vitro and regular blood pressure in vivo. We next analyzed directly the contractility of arterial smooth muscle in isolated arterial rings of WT and $\mathrm{KO}$ mice. Reduced contractility of femoral artery explants from KO mice $(-39.1 \%$, from $4,608 \pm 596 \mu \mathrm{N}$ to $2,793 \pm 214 \mu \mathrm{N} ; n=8-12$ vessels per genotype, $P<0.05)$ was observed after stimulation with $80 \mu \mathrm{M}$ extracellular potassium, which causes cellular depolarization and therefore probes the basic ability for contraction (Figure 4A). To analyze whether KO mice show specific deficits to contractile cues that might act on top of a more unspecific impairment of contraction, we tested the response of artery explants to the two most important regulatory pathways of the vascular tone, adrenergic and angiotensin signaling. We observed a nearly complete loss of the ability of artery explants to contract in response to angiotensin II stimulation and a dramatic deficit after stimulation with the $\alpha_{1}$-adrenoceptor agonist phenylephrine (Figure 4, B-D). We also wanted to know whether the contractile deficit of $\mathrm{KO}$ vessels was due 


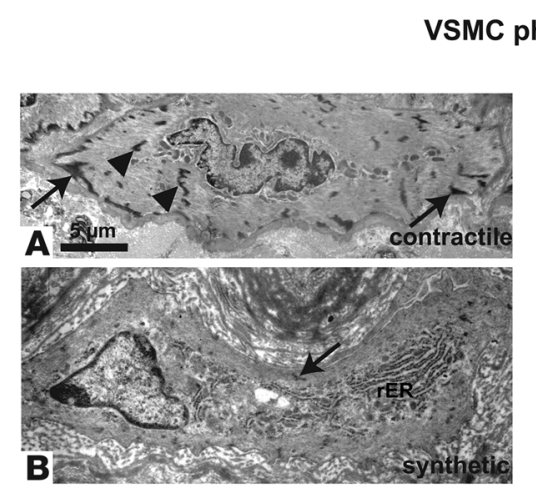

SMC phenotype switch
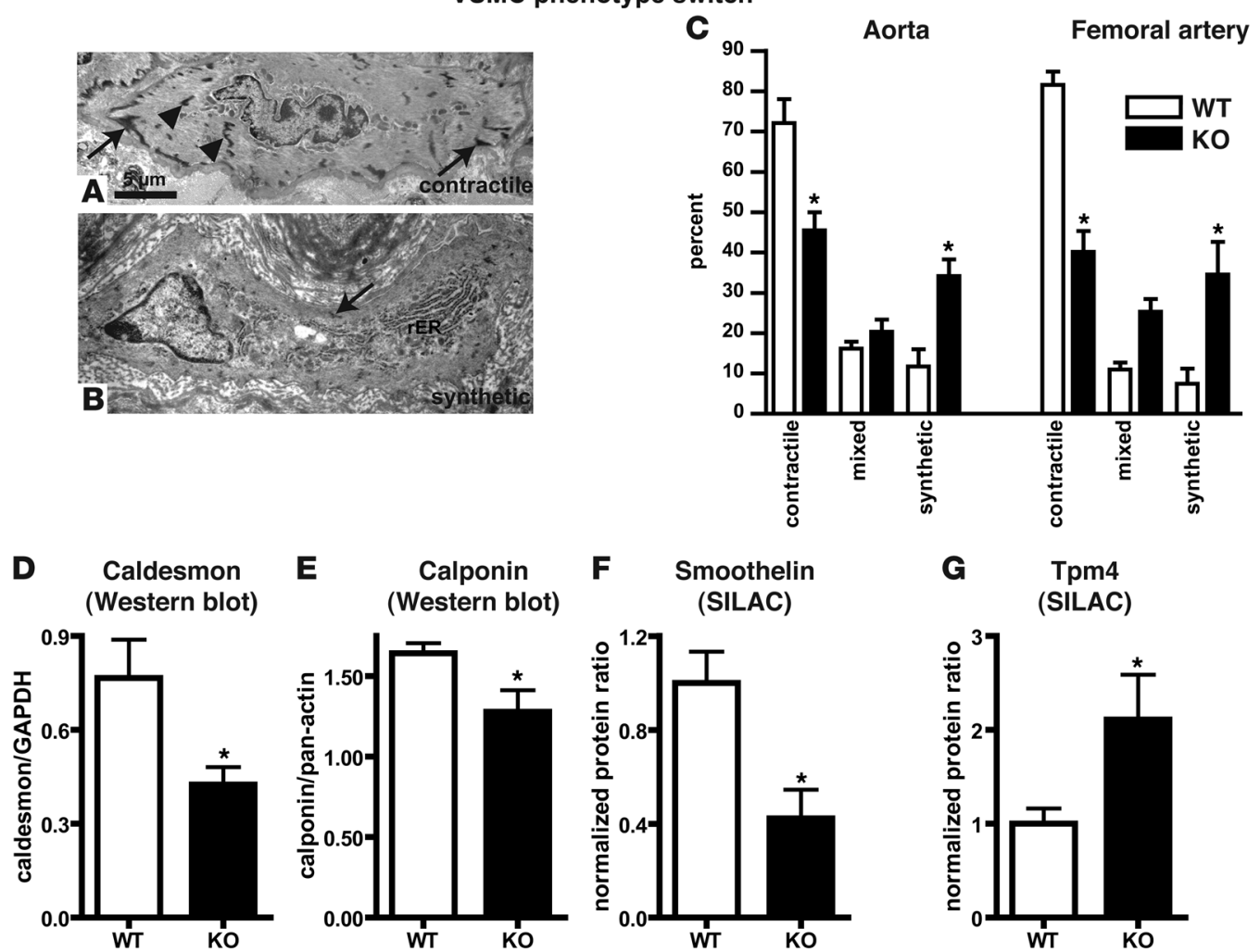
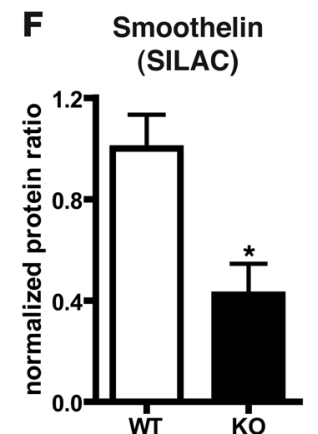

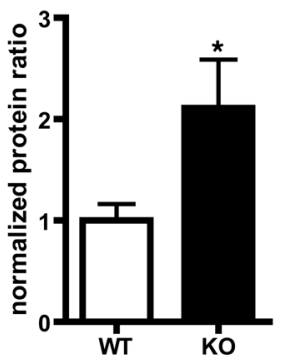

Femoral artery - reduced media size
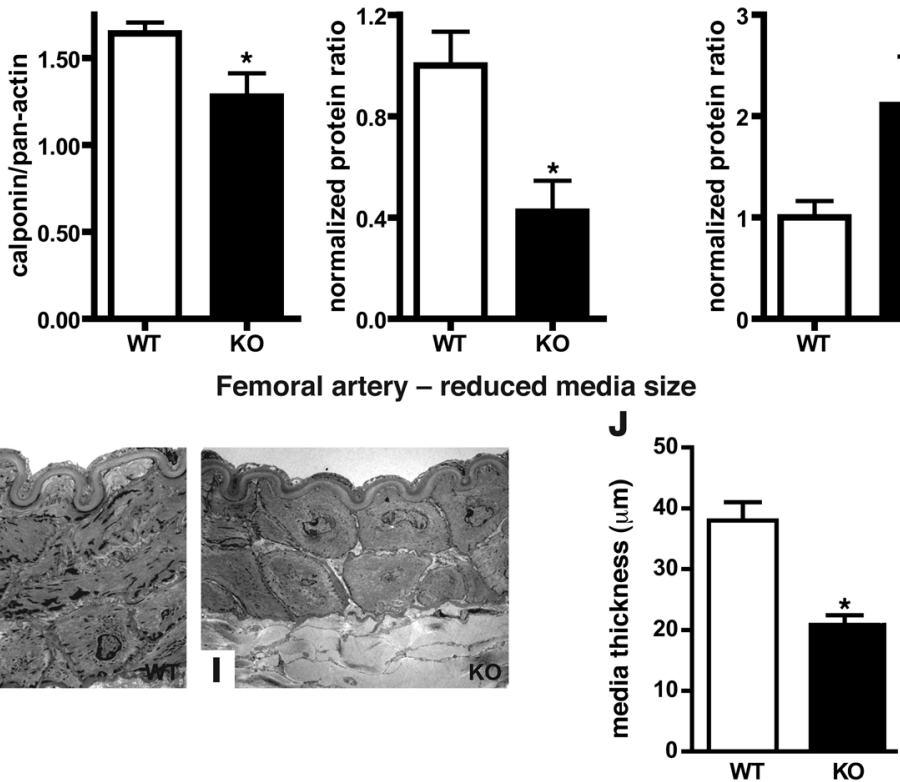

\section{Figure 3}

SMCs of Mir143/145-KO mice show a shift from a contractile to a synthetic phenotype and reduced media thickness. (A and B) Ultrastructural features of contractile and synthetic SMCs. (A) Typical contractile SMCs from WT animals show numerous focal adhesions (arrows) and intracellular dense bodies (arrowheads). (B) In contrast, synthetic SMCs from mutant mice only rarely display focal adhesions (arrow) and intracellular dense bodies and are rich in rough ER ( $r E R)$. (C) Relative numbers of synthetic and contractile VSMCs in aorta and femoral artery. The number of synthetic SMCs is increased in the aorta and femoral arteries of KO mice. ${ }^{*} P<0.05 ; 300$ cells per genotype were analyzed. (D-G) Quantification of markers of contractile SMCs (D-F) and synthetic SMCs (G). ${ }^{*} P<0.05$ in $\mathbf{D}, \mathbf{E}$, and $\mathbf{G} ; n=3$ WT, $n=3 \mathrm{KO}$ mutant. ${ }^{\star} P<0.0001$ in $\mathbf{F}, n=8 \mathrm{WT}, n=7 \mathrm{KO}$ mutant. (H and I) EM pictures of cross sections through the femoral artery. Note the presence of contractile and synthetic SMCs in WT and mutant vessel walls, respectively. (J) Quantification of media thicknesses. ${ }^{*} P<0.05, n=\mathrm{WT}, n=3 \mathrm{KO}$ mutant. Error bars indicate \pm SEM.

to lower $\mathrm{Ca}^{2+}$ sensitivity of the myofilaments and/or to altered receptor signaling. We therefore permeabilized femoral artery segments with Triton $\mathrm{X}-100$ and measured $\mathrm{pCa}^{2+}$-force relationships (Figure 4F) $(13,14)$. Maximal $\mathrm{Ca}^{2+}$-induced vasoconstriction was significantly blunted in $\mathrm{KO}$ vs. WT vessels $(-31 \%$, WT: $1,228 \pm 92 \mu \mathrm{N}, \mathrm{KO}: 846 \pm 88 \mu \mathrm{N} ; n=8-12$ vessels per genotype, $P<0.05)$. Furthermore, the $\mathrm{pCa}^{2+}$ curves were shifted leftward in $\mathrm{KO}$ vs. WT femoral arteries ( $\mathrm{pEC}_{50}, \mathrm{KO}: 6.113 \pm 0.005$, WT: $5.785 \pm 0.007$; $P<0.001)$, indicating that $\mathrm{Ca}^{2+}$ sensitivity was slightly increased in $\mathrm{KO}$ vessels. In principle, the contractile deficit of $\mathrm{KO}$ vessels might be caused by the accumulation of synthetic SMCs, which are unable to develop the same force as contractile SMCs; by a general defect in all SMCs; or by a combination of both. To distinguish between these possibilities, we normalized maximal vasoconstriction effects to the number of contractile cells in the medium. Interestingly, normalized contractile force elicited by $\mathrm{Ca}^{2+}$ in skinned vessels or by $\mathrm{K}^{+}$depolarization in intact vessels did not differ between WT and Mir143/145 mutant mice $(n=8-12$ 


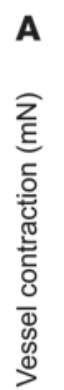

$\mathrm{K}^{+}$- induced contraction

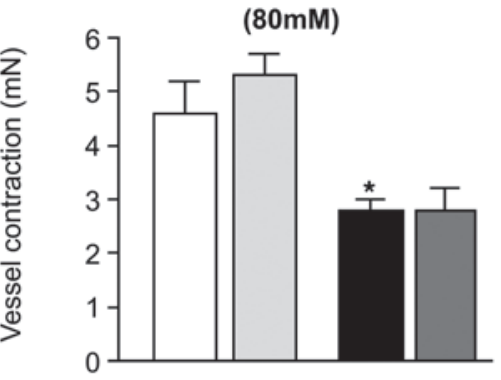

miR-143/145 +/+ +/+ $\quad-/-\quad$ - $/-$ chronic ACE - + - + inhibition

\section{Phenylephrine - induced contraction}

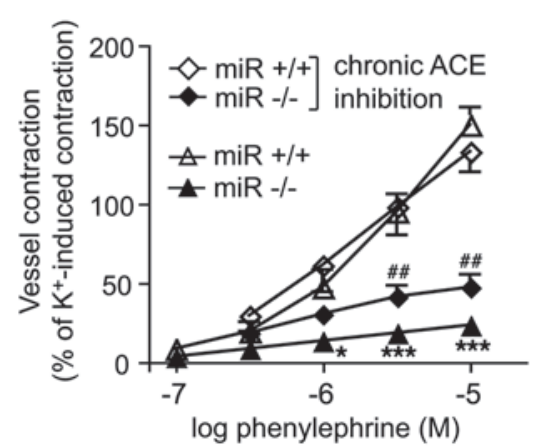

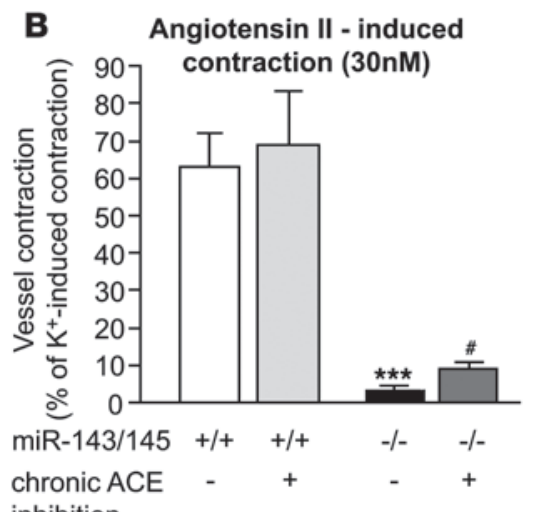

inhibition

E

\section{Phenylephrine - induced contraction}

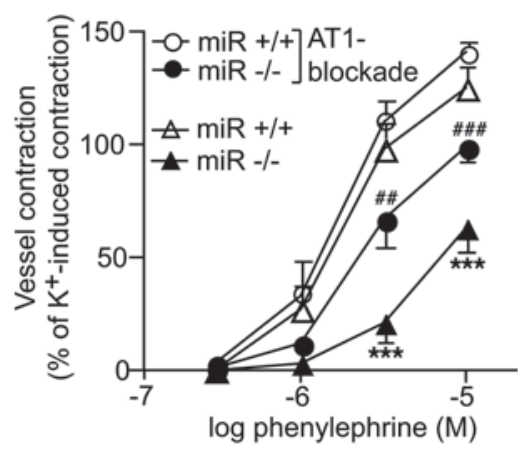

\section{Phenylephrine - induced}

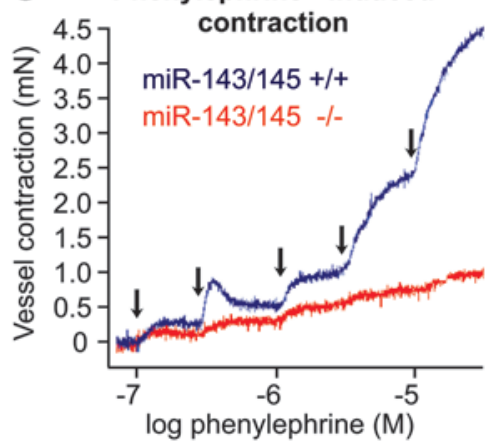

F

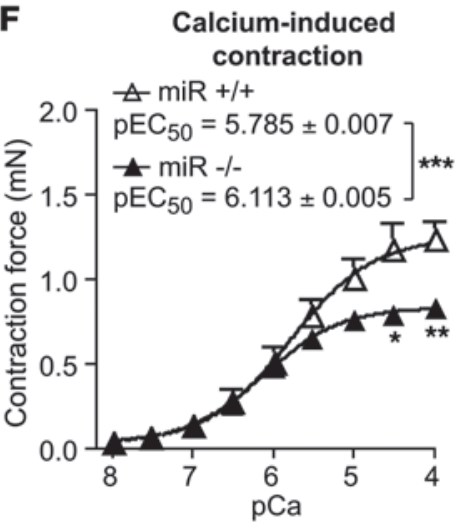

Figure 4

In vitro evaluation of contractile properties of isolated femoral artery rings mounted in a myograph. (A) Vessel contraction induced by extracellular potassium was reduced to $61 \%$ of the WT response $\left(n=12 \mathrm{WT}\right.$ and $n=8 \mathrm{KO}$ vessels, $\left.{ }^{*} P<0.05\right)$. No change in contraction after potassium-induced depolarization was observed after treatment with captopril ( $n=8 \mathrm{WT}$ and $n=9 \mathrm{KO}$ vessels). (B) Vessel contraction induced by angiotensin II was significantly blunted in mutant arteries. Pretreatment with captopril significantly improved angiotensin II-induced contractile responses ( $n=12 \mathrm{WT}$ and $n=9 \mathrm{KO}$ vessels of untreated animals, ${ }^{* * *} P<0.0001$, KO untreated vs. WT untreated; $n=8 \mathrm{WT}$ and $n=9 \mathrm{KO}$ vessels of captopril-treated animals, ${ }^{\#} P<0.05 \mathrm{KO}$ treated vs. KO untreated). (C and D) Isolated arteries stimulated with increasing concentrations of phenylephrine. Arrows indicate applications of phenylephrine. (D) Statistical analysis of phenylephrine and captopril responses. Captopril treatment improved responses of the KO vessels $\left(n=12\right.$ WT and $n=8 \mathrm{KO}$ vessels of untreated animals, ${ }^{*} P<0.05,{ }^{* \star \star} P<0.001, \mathrm{KO}$ untreated vs. WT untreated; $n=8 \mathrm{WT}$ and $n=0 \mathrm{KO}$ vessels of captopril-treated animals, ${ }^{\#} P<0.01 \mathrm{KO}$ treated vs. KO untreated). (E) Contractile responses to phenylephrine stimulation of mesenteric arteries. Arterial rings from $\mathrm{KO}$ animals showed a significant enhancement in contractility after $\mathrm{AT}_{1}$ receptor blockade by losartan $\left(n=7 \mathrm{WT}\right.$ and $n=8 \mathrm{KO}$ vessels from untreated animals, ${ }^{\star \star \star} P<0.001, \mathrm{KO}$ untreated vs. WT untreated; $n=10$ WT and $n=8 \mathrm{KO}$ vessels from losartan-treated animals, \#\# $P<0.01$, \#\#\# $P>0.001$, KO treated vs. KO untreated). (F) pCa-force relationships in skinned femoral arteries. Maximal $\mathrm{Ca}^{2+}$-induced contraction was blunted in $\mathrm{KO}$ arteries, and the $\mathrm{pEC} \mathrm{C}_{50}$ values significantly shifted leftward in $\mathrm{KO}$ skinned arteries ( $n=12 \mathrm{WT}$ and $n=8 \mathrm{KO}$ vessels, ${ }^{*} P<0.05,{ }^{* \star} P<0.01,{ }^{* \star *} P<0.001, \mathrm{KO}$ vs. WT). Error bars indicate \pm SEM.

vessels per genotype, $P<0.05)$. However, normalized receptormediated responses induced by angiotensin II and phenylephrine were severely reduced ( $95 \%$ for angiotensin II and $85 \%$ for phenylephrine) in KO vessels ( $n=8-12$ vessels per genotype; $P<0.01$ for angiotensin II and $P<0.001$ for phenylephrine), suggesting that the lack of miR-143/145 impairs receptor-mediated responses in contractile SMCs. Of course, defects in receptor-mediated contraction of remaining contractile VSMCs is almost certainly only one reason for the impaired contractility of vessels in Mir143/145-KO mice. The presence of synthetic SMCs, which show a major contractile defect, in mutant vessels will also contribute to the contractile deficit of KO vessels. Taken together, our results demonstrate that multiple mechanisms, which include accumulation of synthetic VSMCs and the inability of remaining contractile VSMCs to contract in response to receptor-mediated signals, contributed to reduced contractility of artery explants.
We reasoned that the morphological alterations of VSMCs and decreased contractility of arteries might also affect the vascular tone of arteries in mutant animals in vivo. Intravascular blood pressure measurements disclosed reduced systolic and diastolic blood pressure in mutant compared with control animals under anesthesia (systolic pressure, WT, $n=6: 100 \pm 5.5 \mathrm{mmHg}$ vs. KO, $n=6: 86 \pm 2.4 \mathrm{mmHg}, P<0.05$; diastolic pressure, WT, $n=6$ : $70 \pm 7.0 \mathrm{mmHg}$ vs. KO, $n=6: 49 \pm 4.4 \mathrm{mmHg}, P<0.05)$. To avoid potential artifacts caused by anesthesia, we also measured blood pressure telemetrically using catheters implanted into the femoral artery. Again, systolic blood pressure was significantly reduced in $\mathrm{KO}$ animals, while the heart rate was not altered (Figure 5, A and B). Intravenous application of angiotensin II resulted in a compromised increase in blood pressure in KO compared with WT mice (Figure 5C). To test whether the reduced pressure response was due to desensitization of VSMCs by increased angiotensin II 
A

Blood pressure

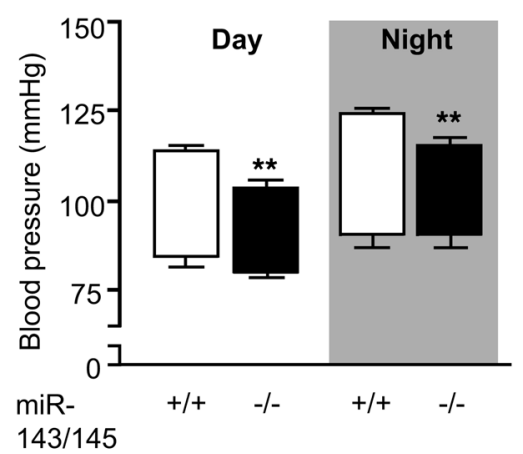

\section{Angiotensin II - induced} hypertension

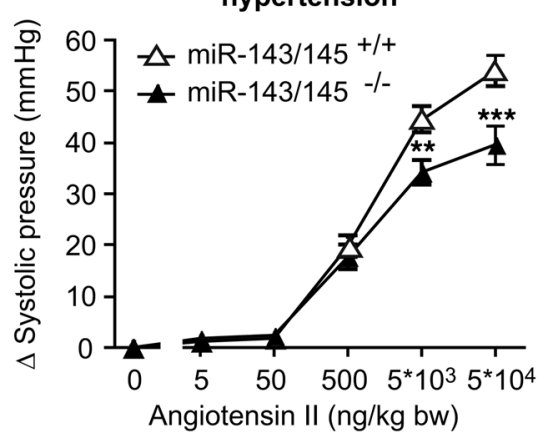

B

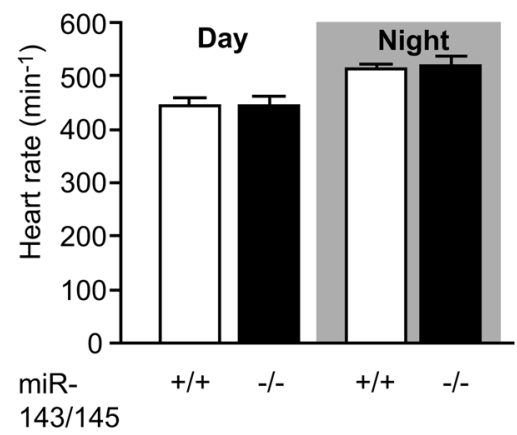

D Acute ACE inhibition - induced

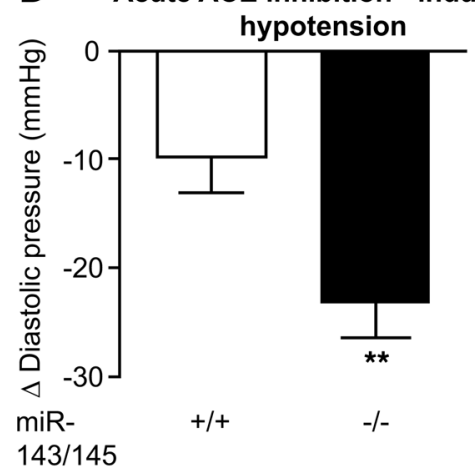

\section{Figure 5}

Hemodynamic measurements. (A and $\mathbf{B}$ ) In vivo blood pressure and heart rate measurements by telemetry. (A) Boxes show blood pressure amplitudes, with upper line representing systolic pressure and lower line, diastolic pressure; error bars indicate SEM. Systolic blood pressure was significantly reduced in miR-143/145-deficient mice during day- and nighttime periods ( $n=6 \mathrm{WT}$ and $n=7 \mathrm{KO},{ }^{* \star} P<0.01$ ), while diastolic pressure did not differ significantly between genotypes. (B) No difference in heart rate was observed between genotypes. (C) During isoflurane anesthesia, angiotensin II induced a larger increase in systolic blood pressure in WT $(n=12)$ than in KO mice $(n=13)\left({ }^{* *} P<0.01,{ }^{* \star} P<0.001\right)$. (D) Acute inhibition of ACE by captopril lead to a greater decrease in diastolic pressure in miR-143/145deficient animals $(n=11 \mathrm{WT}$ and $n=13 \mathrm{KO}$, $\left.{ }^{* *} P<0.01\right)$. Error bars indicate \pm SEM. signaling in vivo, we blocked the formation of angiotensin II by administration of captopril. Acute inhibition of angiotensin-converting enzyme (ACE) led to a pronounced reduction in diastolic pressure in KO animals, which corresponded to the increased angiotensin II signaling (Figure 5D). We also detected a reduced cardiac ventricle/body weight ratio (WT, $n=12: 4.93 \pm 0.09 \mathrm{mg} / \mathrm{g}$ vs. KO, $n=14: 4.44 \pm 0.12 \mathrm{mg} / \mathrm{g} ; P<0.01)$ and a reduced ventricle weight/tibia length ratio (WT, $n=12: 8.47 \pm 0.24 \mathrm{mg} / \mathrm{mm}$ vs. $\mathrm{KO}, n=14: 7.67 \pm 0.27 \mathrm{mg} / \mathrm{mm} ; P<0.05)$, probably as a result of the reduced blood pressure, while body weights did not differ between Mir143/145 mutant and WT mice. To rule out a potential contribution of other SMC-containing organs (lung, gastrointestinal tract) to the hypotensive phenotype, we examined a number of additional physiological parameters. (a) Analysis of blood gas composition did not uncover changes in $\mathrm{pO}_{2}, \mathrm{pCO}_{2}$, base excess of extracellular fluid (BEecf), $\mathrm{HCO}_{3}, \mathrm{TCO}_{3}$, or $\mathrm{sO}_{2}$ (WT: $n=5, \mathrm{KO}$ : $n=5$, data not shown), indicating that the pulmonary gas exchange was not compromised in KO mice. (b) No signs of cardiac malfunctions or RV hypertrophy secondary to changes in pulmonary function (mean RV myocyte cross-sectional area: WT, $n=6$ : $214.1 \pm 14.4 \mu \mathrm{m}^{2}$ vs. KO, $n=7: 221.2 \pm 17.5 \mu \mathrm{m}^{2}$ ) were noted. (c) Transit time through the gastrointestinal tract was unaltered (WT, $n=6: 250 \pm 21$ minutes vs. KO, $n=6: 260 \pm 16$ minutes).

miR-143 and miR-145 modulate regulators of SMCs at different levels. To elucidate the molecular changes that resulted in the impairment of the contractile phenotype in VSMCs of miR-143/145-KO animals, we utilized a high-throughput approach developed by our group. Our experimental system was based on quantitative, mass spectrometry-based (MS-based) proteomics using reference mice that were completely labeled with a ${ }^{13} \mathrm{C}_{6}$-lysine-substituted version of lysine ("SILAC mice") (15), combined with microarray- based transcriptional profiling. SILAC analysis of aortae lacking expression of miR-143/145 ( $n=3$ per genotype) was employed to determine the relative changes of approximately 1,600 proteins in mutant mice relative to WT controls (Figure 6, A-E, and Supplemental Tables 1 and 2). The SILAC approach was corroborated by Western blot analysis (Figure 6H and Supplemental Figure 2) and immunofluorescence staining (Figure 6, F and G) of selected proteins. In addition, we analyzed mRNA expression profiles of aortae from KO and WT animals using Affymetrix GeneChips ( $n=5$ per genotype) to identify possible similarities and differences between changes in protein and mRNA levels in KO and WT animals (Supplemental Tables 3 and 4). Since miRNAs affect not only the stability of mRNAs but also the efficiency of protein translation, this approach allowed us not only to identify the maximum number of potentially affected proteins but also to gain an idea about the mechanism involved.

Transcripts and proteins, which differed significantly between WT and KO tissues, were matched to potential target transcripts predicted by the miRanda database (16) (http://www.microRNA. org) (17) and miRBase (18) or TargetScan 4.2 algorithm (19) for miR-143 and miR-145 using the MatchMiner tool (20). For the analysis, we omitted those transcripts that were normalized by extended treatment with the ACE inhibitor captopril or the angiotensin II type $1\left(\mathrm{AT}_{1}\right)$ receptor inhibitor losartan (see below), since they are unlikely to represent primary targets of miR-143/145. We found an upregulation of miR-143/145 mRNA targets that are known to play important roles in the biology of SMCs (miR-145 target: Argagp12; Tpm4 [tropomyosin 4] is target of both miR-143 and miR-145) (1). In addition, several other mRNAs were increased that fulfill tasks in various other cell types but lack an apparent specific function in VSMCs (Supplemental Table 5A). 
A WT (labeled) versus WT (nonlabeled)

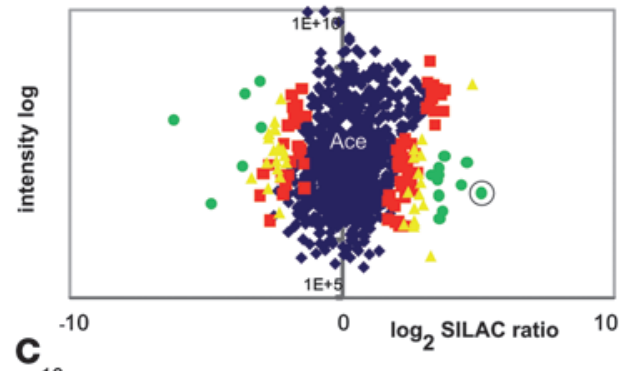

C

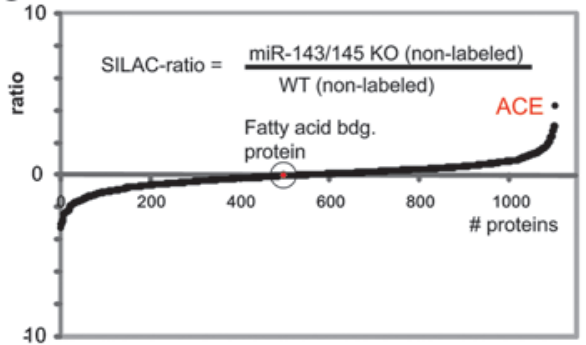

D

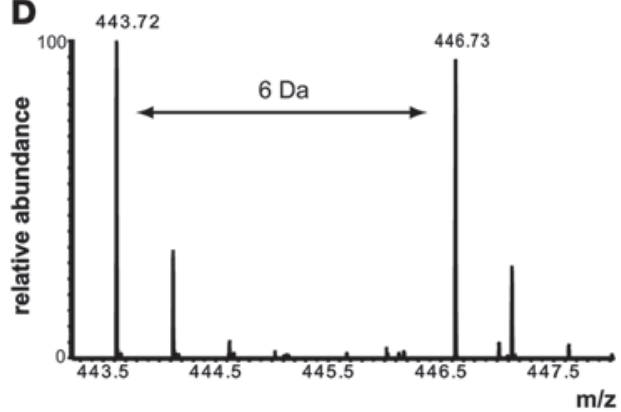

$\mathbf{F}$

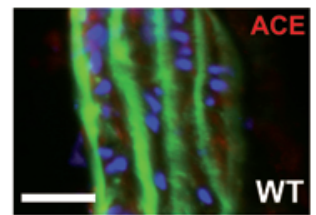

G

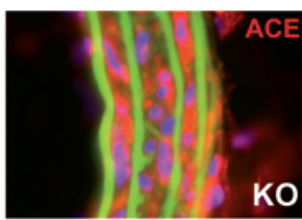

I

3. UTR Mouse ACE (ENSMUST00000001963)

3' uuCCCUA-AGG--ACCCUUUUGACCUg 5' Score: 151

|| : | ||| | | |||||||| 19.9

29 gaGGAGTGTCCCATAAG-AAACTGGAt 54

$3^{\prime}$ uuCCCUAAGGACCCUUUUGACCUg 5' Score: 111

|II| || || :|| ||| Energy: -21.2

250 gtGGGAAGCCAGG---GACAGGAC 270

3. uuCCCUAAGGACCCUUUU----GACCUg 5'

III $11: 11|:: 1|$ IIIII
557 ctGGGTTCTTGGAGGAAGGCTCTGAT 584

Score: 118

Energy: -26.0

4. exon mouse ACE (ENSMUST00000001963)

3. uucCCUAA-GGACCCUUUUGACCug 5 '

| ||||||||||$:::|||| \mid$
604 tgttGTTTGCCTGGGAGGGTGGCa 628

Score: 128

Energy: -29.0

E
B WT (labeled) versus miR-143/145 KO (nonlabeled)
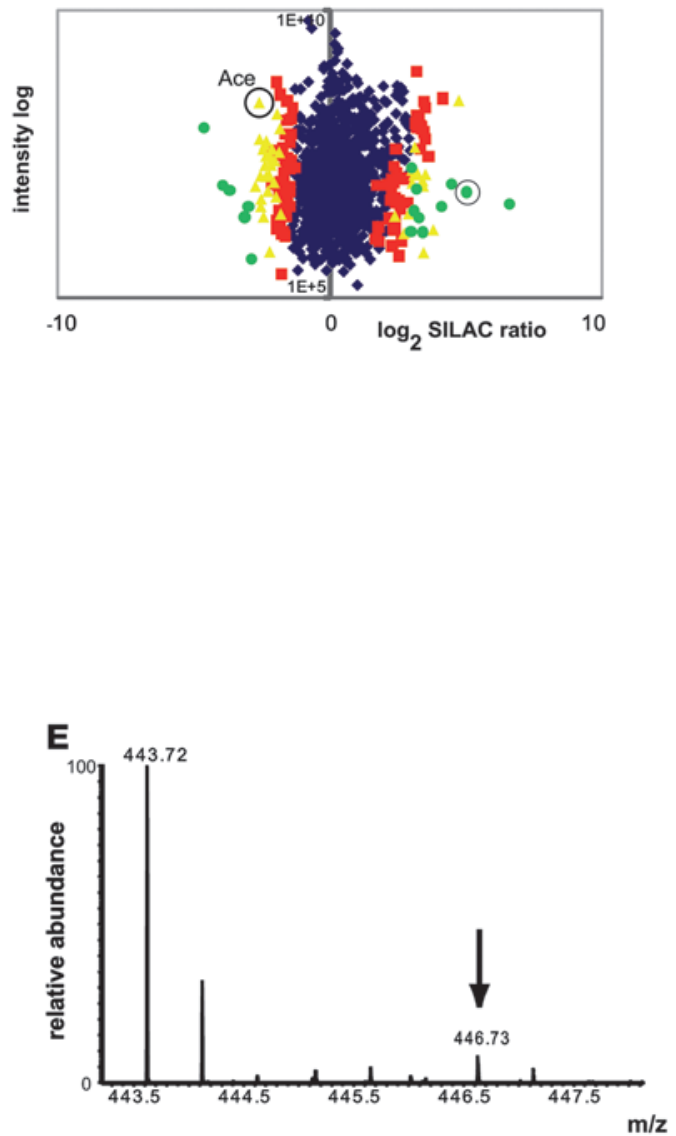

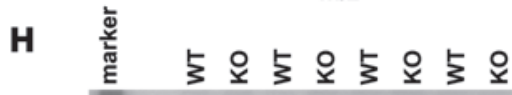

220 kDa

$120 \mathrm{kDa}$

100 kDa

ACE-1

$40 \mathrm{kDa}$

GAPDH

J

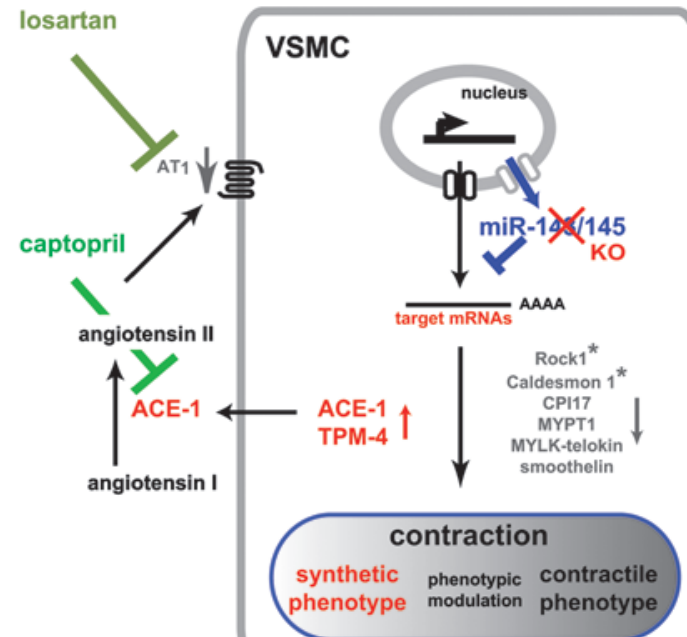




\section{Figure 6}

ACE is a target of miR-145. Analysis of protein expression by SILAC mouse-based quantitative proteomics and Western blot analysis. (A and B) SILAC-labeled $\left({ }^{13} \mathrm{C}_{6}\right.$ Lys) WT aorta was mixed with nonlabeled WT aorta (A) and miR-143/145 KO aorta (B). Protein ratios are plotted against added peptide intensities. Blue dots represent proteins that do not show a significant change in expression levels $(P \geq 0.05)$. Red, yellow, and green dots represent proteins with a significant change in expression levels at different confidence intervals (red: $P<0.05$, yellow: $P<0.01$, green: $P<0.001$ ). Circled green dots in $\mathbf{A}$ and $\mathbf{B}$ indicate fatty acid-binding protein. (C) To obtain ratios between nonlabeled WT and nonlabeled Mir143/145-KO aortae, both ratios from ${ }^{13} \mathrm{C}_{6}$ Lys-WT/WT (nonlabeled) and ${ }^{13} \mathrm{C}_{6}$ Lys-WT/miR-143/145 KO (non-labeled) were divided by each other. (D and E) Mass spectra of an ACE-1 peptide [YVEFSNK (MH2+)] SILAC pair with ${ }^{13} \mathrm{C}_{6}$ Lys-WT/WT (D) and a pair ${ }^{13} \mathrm{C}_{6}$ Lys-WT/Mir143/145-KO (E). Peaks with lower molecular weight correspond to the unlabeled peptide; peaks with higher molecular weight (greater than $6 \mathrm{Da}$ ) correspond to the ${ }^{13} \mathrm{C}_{6} \mathrm{Lys}$ peptide. Calculation of ACE-1 signals from all samples revealed a 4.9-fold upregulation of ACE-1 in KO vs. WT aortae ( $n=3$ per genotype, $P<0.01$; see Supplemental Table 1). (F and $\mathbf{G}$ ) Immunofluorescence analysis of ACE expression in cryosections of aorta (scale bar: $25 \mu \mathrm{m}$ ). (H) Western blot analysis of ACE-1 expression in WT and Mir143/145-KO aortae $(n=4$ per genotype, $P<0.001$, KO/WT ratio: 4.3). (I) miR-145 binding sites in the 3' UTR and ORF of ACE predicted by the miRanda algorithm. (J) Schematic representation of the role of miR-143/145 in VSMCs.

Our unbiased protein analysis did reveal the rise of predicted targets of miR-143/145, such as Tpm4 (Supplemental Table 5B), that were also increased at the mRNA level. As expected, we also detected the upregulation of putative target proteins that were not matched by transcriptional changes (Supplemental Table 5). Most likely these proteins were controlled by miR-143/145-mediated translational repression. Interestingly, one of the most strongly affected proteins in this group was ACE, which converts circulating angiotensin I into its active form angiotensin II (Figure 6J). The $3^{\prime}$ UTR and the ORF of mouse ACE contain several miR-145 binding sites with high scores and low energy as predicted by the miRanda algorithm (16) (Figure 5I). To test the functional significance of the putative miR-145 target sites in the $3^{\prime}$ UTR, we employed a luciferase reporter assay. We found that intact but not mutant miR-145 target sites are able to mediate repression of reporter gene activity upon overexpression of miR-145 (Supplemental Figure 5). The miRanda algorithm also detected miR-145 binding sites in the UTR and ORF of ACE mRNA of other species such as chickens, rats, and humans. Angiotensin II is not only a potent agonist for the contraction of VSMCs but also a major regulator of the contractile phenotype of VSMCs, which explains - at least in part - the shift from the contractile to the synthetic phenotype in Mir143/145-KO mice. Most likely, this mechanism synergizes with effects of miR-143/145 on other targets that are upregulated in $\mathrm{KO}$ mice. In line with this premise, we observed additional changes in the transcript and/or protein expression level of molecules known to influence the SMC phenotype (Supplemental Tables 1-4). TPM-4, which is a predicted target of both miR-143 and miR-145, is a structural protein that is specifically upregulated in synthetic SMCs (21).

The upregulation of ACE in VSMCs of mutant mice raised the question of whether the local rise in ACE concentrations also suffices to increase systemic angiotensin II levels. Interestingly, the level of blood circulating angiotensin II was not upregulated in $\mathrm{KO}$ vs. WT (KO: $16 \pm 3$, WT: $28 \pm 6 \mathrm{fmol} / \mathrm{ml}$ plasma (from $n=9$ $\mathrm{KO}, n=8 \mathrm{WT}$ animals, pooled to $n=4$ and $n=5$ plasma sam- ples, respectively), which might explain phenotypic differences of miR-143/145 mutant mice compared with other transgenic models that rely on the unrestricted overexpression of components of the renin-aldosterone-angiotensin system (RAAS).

In addition to the upregulation of predicted targets, we observed changes in the expression of numerous mRNAs and proteins, which occurred most likely due to secondary events. We detected a downregulation of, among others, angiotensin receptor 1 (Agtr1b), which is a typical response to excessive angiotensin II signaling. The downregulation of angiotensin receptor 1 corresponded to reduced cGMP concentrations in the aortae of KO compared with WT mice (KO, $n=3: 30.0 \pm 6.7$; WT, $n=3: 51.1 \pm 7.9 \mathrm{pmol}$ cGMP/g wet weight). Angiotensin II is known to stimulate cGMP production, an effect that was apparently blocked due the downregulation of angiotensin $\mathrm{AT}_{1}$ receptor. We also found reduced expression of the Agtr1-interacting molecules Freq/NCS-1 (Western blot, Supplemental Figure 2; RNA, Supplemental Table 4) and Kcnd3-KV4.3 (RNA analysis) (22) and of molecules that control the trafficking of plasma membrane receptors such as Cav-2 (SILAC, Supplemental Table 2) and Cav-3 (Western blot, Supplemental Figure 2). In contrast, cholecystokinin and gastrin-releasing peptide (Supplemental Table 3), which are involved in the regulation of contractility of SMCs, were upregulated. In principle, many elements of signaling cascades that govern contraction and migration of SMCs were changed, including a downregulation of RGS4, RGS5, RGS7bp (Supplemental Table 4) and upregulation of the RGS-interacting molecule GNB5, as well as of components of the Rho signaling cascade (Rock1, Rnd2/3, Cdc42ep3, Arggef17) and molecules that direct calcium handling and signaling (SERCA, caldesmon-1, Camk2g) of VSMCs. The effects on the Rho signaling cascade might be of particular significance, since it affects nuclear translocation and/or activation of SRF (23). In addition, several molecules (CPI-17, MYLK-Telokin, MYPT1; Supplemental Tables 2 and 4) were repressed that directly regulate contraction of VSMCs by controlling phosphorylation of myosin light chain. We did not detect changes in the expression levels of $\alpha$-SMA and smooth muscle myosin heavy chain according to our in vivo SILAC measurements. Interestingly, however, we noted a loss the expression of several other markers of differentiated SMCs and an increase in the expression of skeletal muscle markers (Supplemental Tables 2-5).

$A C E$ inhibition partially rescues loss of miR-143/145. To directly test the relevance of the proposed miR-145 target ACE for the observed phenotype, we chronically inhibited ACE and the $\mathrm{AT}_{1}$ receptor by oral administration of captopril and losartan, respectively. According to our model, the lack of ACE repression in Mir143/145-KO animals causes a local increase in angiotensin II signaling in SMCs, which should be rescued by pharmacological inhibition of ACE. We found that a 20-day treatment with captopril markedly improved the contractile response of mutant but not of WT arteries to phenylephrine and angiotensin II (Figure 4, B and D). Maximal angiotensin II-induced force of contraction was $3.2 \pm 0.7$ $\mathrm{mN}$ in untreated WT vessels as compared with $3.4 \pm 0.6 \mathrm{mN}$ in captopril-treated WT vessels $(P=0.84, n=12$ untreated and $n=8$ treated WT vessels). In femoral arteries from $\mathrm{KO}$ mice, contractile force was greater after captopril treatment $(0.30 \pm 0.05 \mathrm{mN})$ than that in untreated $\mathrm{KO}$ specimens $(0.11 \pm 0.03 \mathrm{mN} ; P=0.007$, $n=9$ treated and $n=9$ untreated $\mathrm{KO}$ vessels). While the absolute functional improvement after ACE inhibition was significant but relatively small for angiotensin II-induced contraction $\left(9 \%\right.$ of $\mathrm{K}^{+}$ contraction; Figure 4B), phenylephrine effects increased to $46 \%$ of 
Table 1

Treatment of mutant mice with the ACE inhibitor captopril or the AT1 inhibitor losartan normalizes expression of numerous dysregulated transcripts

\begin{tabular}{|c|c|c|c|c|c|c|c|}
\hline \multirow{2}{*}{$\begin{array}{l}\text { Affymetrix } \\
\text { probe set ID }\end{array}$} & \multirow[t]{2}{*}{ Gene symbol } & \multicolumn{2}{|c|}{ Mir143/145-KO vs. WT } & \multicolumn{2}{|c|}{ Mir143/145-KO captopril vs. WT } & \multicolumn{2}{|c|}{ Mir143/145-KO Iosartan vs. WT } \\
\hline & & FC & $P$ & FC & $\boldsymbol{P}$ & FC & $P$ \\
\hline 1417464_at & Tnnc2 & 5.57 & 0.028 & 1.13 & 0.699 & 1.51 & 0.309 \\
\hline 1427735_a_at & Acta1 & 4.74 & 0.023 & 0.87 & 0.711 & 0.76 & 0.289 \\
\hline 1448756_at & S100a9 & 3.48 & 0.038 & 0.78 & 0.643 & 1.64 & 0.246 \\
\hline 1419312_at & Serca1 & 3.36 & 0.017 & 0.72 & 0.145 & 0.82 & 0.294 \\
\hline 1419394_s_at & S100a8 & 3.16 & 0.042 & 0.81 & 0.636 & 1.83 & 0.108 \\
\hline 1448371_at & Mylpf & 2.75 & 0.027 & 0.77 & 0.223 & 1.05 & 0.759 \\
\hline 1422926_at & $M c 2 r$ & 2.68 & 0.001 & 1.23 & 0.370 & 0.70 & 0.198 \\
\hline 1450468_at & Myoc & 2.13 & 0.001 & 0.99 & 0.940 & 0.76 & 0.309 \\
\hline 1419871_at & Ppp2r1b & 1.90 & 0.030 & 0.81 & 0.331 & 0.65 & 0.080 \\
\hline 1420647_a_at & Krt8 & 1.78 & 0.022 & 1.09 & 0.686 & 1.00 & 0.992 \\
\hline 1433486_at & Clcn3 & 1.73 & 0.003 & 1.09 & 0.474 & 1.27 & 0.085 \\
\hline 1427183_at & Efemp1 & 1.71 & 0.005 & 1.22 & 0.379 & 0.71 & 0.182 \\
\hline 1433694_at & Pde3b & 1.71 & 0.037 & 1.04 & 0.894 & 0.87 & 0.548 \\
\hline 1435184_at & Npr3 & 1.61 & 0.017 & 1.00 & 0.996 & 1.68 & 0.035 \\
\hline 1455158_at & Itga3 & 0.48 & 0.002 & 0.76 & 0.053 & 0.84 & 0.259 \\
\hline 1440789_at & Neo1 & 0.49 & 0.002 & 1.07 & 0.654 & 1.27 & 0.084 \\
\hline 1423222_at & Cap2 & 0.52 & 0.003 & 0.77 & 0.151 & 1.36 & 0.072 \\
\hline 1449522_at & Unc5c & 0.41 & 0.005 & 0.63 & 0.112 & 0.80 & 0.281 \\
\hline 1424768_at & Cald1 & 0.50 & 0.008 & 1.09 & 0.515 & 1.53 & 0.002 \\
\hline 1460729_at & Rock1 & 0.45 & 0.009 & 0.99 & 0.944 & 1.77 & 0.050 \\
\hline 1423942_a_at & Camk2g & 0.58 & 0.011 & 0.87 & 0.362 & 1.17 & 0.490 \\
\hline 1433682_at & Arhgef17 & 0.63 & 0.016 & 0.82 & 0.210 & 1.11 & 0.476 \\
\hline 1449876_at & Prkg1 & 0.45 & 0.017 & 1.12 & 0.671 & 1.16 & 0.545 \\
\hline 1425506_at & Mylk & 0.70 & 0.026 & 0.90 & 0.202 & 1.06 & 0.598 \\
\hline
\end{tabular}

Affymetrix GeneChip analysis revealed 507 probe sets that were significantly changed between WT and $\mathrm{KO}(P<0.05 ; \mathrm{FC}>1.5$ or $\mathrm{FC}<0.666 ; n=5$ per genotype). Of these probe sets, 248 showed WT levels after chronic ACE inhibition for 20 days $(P>0.05, n=3)$. Of the 507 probe sets, 220 were not significantly changed after 20 days of losartan treatment $(P>0.05, n=3)$. Losartan and captopril both suppress changes induced by upregulated expression of the miR-145 target ACE. Therefore, the effects of both inhibitors strongly overlap: expression of 164 probe sets was normalized by both losartan and captopril. Significant differences are indicated in bold.

the $\mathrm{K}^{+}$contraction (Figure 4D) after ACE inhibition. In contrast, the maximal force of contraction of $\mathrm{KO}$ arteries generated by $\mathrm{K}^{+}$induced depolarization did not improve (Figure 4A). Similarly, treatment of $\mathrm{KO}$ mice with the $\mathrm{AT}_{1}$ receptor antagonist losartan for 3 weeks resulted in an enhancement of vasoconstriction of phenylephrine-induced contraction by $214 \%$ or $57 \%$ at $3 \mu \mathrm{M}$ and $10 \mu \mathrm{M}$ phenylephrine, respectively, which corresponds to $96 \%$ of the $\mathrm{K}^{+}$force (Figure 4E). In WT mice, losartan did not affect phenylephrine-induced vasoconstriction (Figure 4E).

Microarray analysis revealed that the partial rescue of agonistinduced contractility was accompanied by normalization of several transcripts, such as Rock1, SERCA, caldesmon-1, and Camk2g (Table 1), that are involved in receptor-mediated contractility of SMCs and calcium signaling. In total, 248 of the 507 probe sets that were deregulated in mutant compared with WT mice $(P<0.05$, fold change $[\mathrm{FC}]>1.5$ or $\mathrm{FC}<0.666, n=5$ mutant and $n=5 \mathrm{WT}$ ) returned to normal levels after 3 weeks of oral application of captopril to mutant mice ( $n=3$ per genotype) (Table 1$)$. Similarly, we observed a normalization of 220 of the 507 probe sets $(P<0.05$, $\mathrm{FC}>1.5$ or $\mathrm{FC}<0.666 ; n=5$ per genotype) after 3 weeks of oral application of the $\mathrm{AT}_{1}$ receptor blocker losartan to mutant mice $(n=3$ per genotype, $P>0.05$; Table 1$)$. We also found that the probe sets, which were normalized by either captopril or losartan, strongly overlapped. There were 164 probe sets common to the group of 248 deregulated in response to captopril and 220 deregulated in response to losartan. Although this experiment clearly indicates the functional relevance of the miR-145 target ACE for the contractile dysfunction, we did not observe significant morphological changes in arteries of $\mathrm{KO}$ mice after 20 days treatment with captopril or losartan (data not shown).

Development of neointimal lesions. Transitions of the phenotypic state of VSMCs play a key role in the repair of vascular injuries and in the development and/or progression of atherosclerosis, which is also reflected by the rapid downregulation of multiple miRNAs during neointima development after vascular injury (24). We therefore turned our focus to long-term changes in the vascular system that might arise as a result of the accumulation of synthetic VSMCs. Importantly, we found neointimal lesions in the femoral arteries of 18-month-old Mir143/145 mutant mice $(n=7)$, which constituted $49.12 \% \pm 5.75 \%$ of the media. Neointimal lesions were absent in WT controls $(n=9)$ and negligible in younger Mir143/145 mutants $(n=8)$ (Supplemental Figure 4). Early lesions in Mir143/145 mutant mice were characterized by the presence of SMCs in the neointima (Figure 7, B, E, and G), while more mature lesions contained large amounts of SMCs and macrophages and deposits of amorphous collagen I, which resulted in the formation of large plaques (Figure 7, C, F, H, K, M, and N). We did not observe lipid-rich cores or foam cells within the lesions, which distinguishes them from classic atherosclerotic plaques. Similarly, the localization of plaques in the femoral arteries and not the aortic 

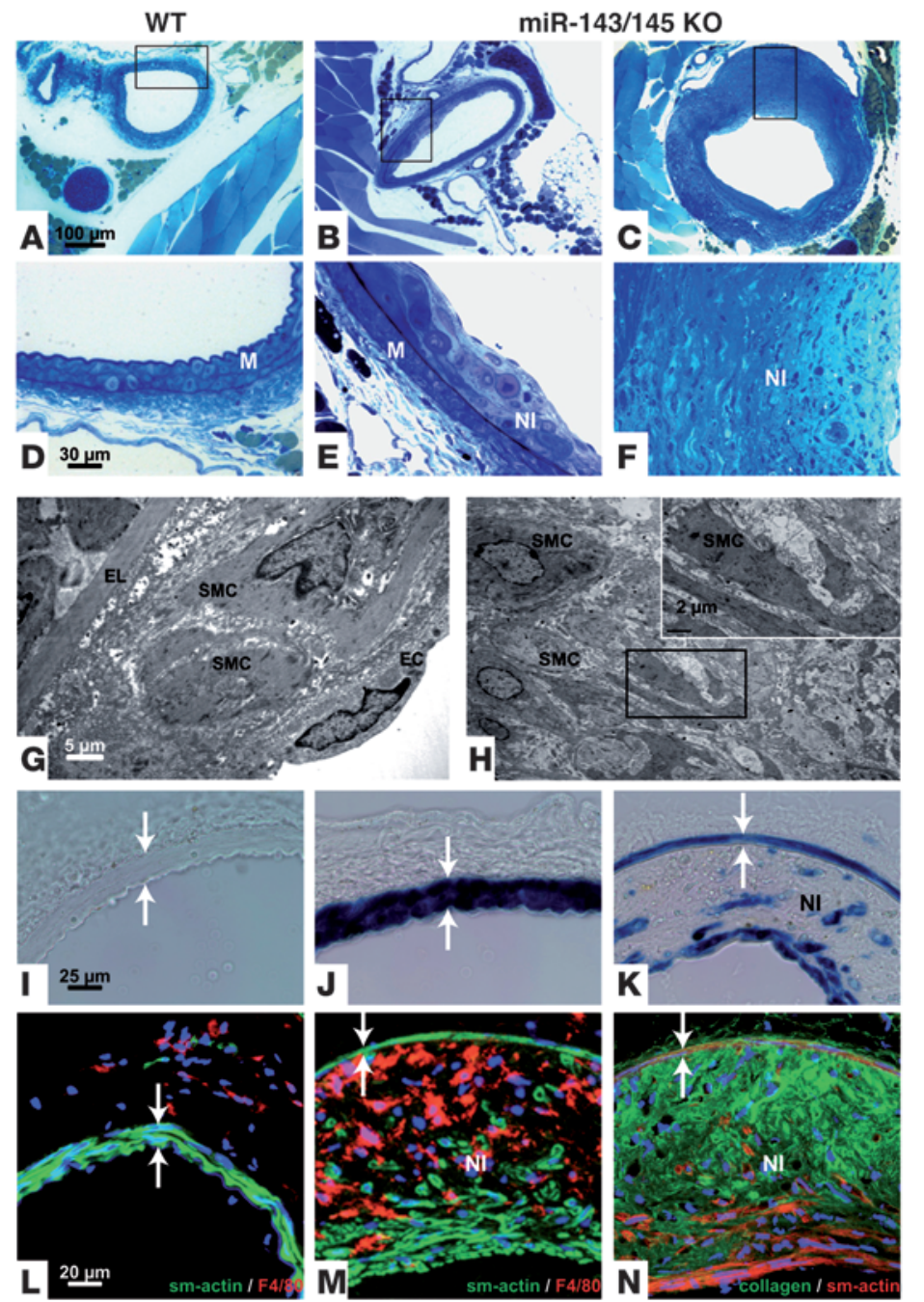

\section{Figure 7}

Mir143/145-KO mice develop neointimal lesions. (A-C) Sections of WT $(\mathbf{A})$ and $\mathrm{KO}(\mathbf{B}$ and $\mathbf{C})$ femoral arteries are shown (scale bar, $100 \mu \mathrm{m}$ ); boxed regions are magnified in $\mathbf{D}-\mathbf{F}$ (scale bar, $30 \mu \mathrm{m})$. Electron micrographs of early and late lesions are shown in $\mathbf{G}$ and $\mathbf{H}$, respectively. Scale bars: $5 \mu \mathrm{m}(\mathbf{G}), 2 \mu \mathrm{m}(\mathbf{H}$, inset). (I-K) Femoral artery of WT (I), heterozygous (J), and homozygous animals $(\mathbf{K})$ stained for $\beta$-galactosidase activity to indicate miR-143/145 locus activity (scale bar, $25 \mu \mathrm{m}$ ). (L and $\mathbf{M}$ ) Sections of $\mathbf{W T}(\mathbf{L})$ and $K O$ vessels (M). Sections of the femoral artery stained with antibodies against $\alpha$-SMA or the macrophage marker F8/40. (N) Sections of the femoral artery stained with antibodies against $\alpha$-SMA and collagen I. Scale bar: $20 \mu \mathrm{m}(\mathbf{L}-\mathbf{N})$. Lesions were frequently found in $\mathrm{KO}$ animals but not in WT animals. (G) Formation of neointima with SMCs and macrophages (not shown) between the lamina elastica and endothelial cells. Lesions at later stages reached large volumes and showed a thinned media (I-N, arrows) but no constriction of the vessel. Note the absence of foam cells or lipophilic inclusions $(\mathbf{G}$ and $\mathbf{H})$, which was also indicated by the absence of Sudan IV staining (not shown). SMCs were recognized by morphology ( $\mathbf{G}$ and $\mathbf{H})$, by anti-smooth muscle staining ( $\mathbf{M}$ and $\mathbf{N}$ ), or by lac $Z$ transgene expression under the control of the miR$143 / 145$ locus (K). The plaques contain macrophages identified by the F4/80 marker (M) and deposits of amorphous collagen (N). Smooth muscle (G) and macrophages were also identified in early lesions (not shown). EL, elastic lamina; NI neointima. Scale bars: $25 \mu \mathrm{m}(\mathbf{I}-\mathbf{K}) ; 20 \mu \mathrm{m}(\mathbf{L}-\mathbf{N})$. arch distinguishes Mir143/145-KO mice from disease models such as apoE-deficient mice and others that are characterized by a massive increase in serum lipoproteins (25). In line with these findings, we found normal serum concentrations of triglycerides and lipoproteins in Mir143/145-KO mice (data not shown).

\section{Discussion}

Our report defines the Mir143/145 gene cluster as a major regulator of the contractile phenotype of VSMCs. We have demonstrated that the miR-143/145 gene cluster governs the expression level of molecules that control the balance between the synthetic and the contractile state of SMCs and directly affect contractility of VSMCs. Such a function fits the widely assumed role of miRNAs as effectors acting in a context-dependent, combinatorial manner $(19,26)$. The restricted expression of miR-143/145 initially in early cardiomyocytes and then in SMCs identifies this miRNA family as a new cell type-specific regulator of SMCs, which enlarges the relatively small group of SMC-specific regulators. Other regulators of SMCs show a less restricted expression profile, e.g., myocardin, which plays a pivotal role as a coregulator of SRF in SMCs but is also expressed in adult cardiomyocytes (27). Our microarray and reporter genebased expression analysis did not reveal major expression sites of miR-143 and miR-145 in other cell types, although we cannot rule out low-level or context-dependent expression patterns. We did not observe defects during early embryogenesis, which is in contrast to the recently reported function of $\mathrm{miR}-145$ as an inducer of lineagerestricted differentiation (28).

In this study, we have used for the first time to our knowledge an unbiased quantitative proteomics approach based on selective labeling of a reference mouse with ${ }^{13} \mathrm{C}_{6}$ Lys ("SILAC-mouse") to analyze the interaction of miRNAs with the transcriptome in vivo. Combination of the proteome analysis with whole-genome transcriptional profiling and bioinformatic target searches allowed us to identify several putative miR-143 and/or miR-145 targets. Although the majority of the putative targets were upregulated at the transcriptional level, we also detected targets that were changed at the posttranscriptional but not on the transcriptional level, which strongly argues for an miRNA-mediated mechanism of regulation. One of the most interesting candidates in this group was ACE, which is a predicted miR-145 target. The ACE cleavage product angiotensin II exerts multiple effects on VSMCs, affecting both their contraction and phenotype $(29,30)$. Under physiological conditions, the widely expressed ACE is only found at low levels in the vasculature. Vascular lesions result in activation of angiotensin II signaling, which together with other factors favors the acquisition of a synthetic phenotype (31). We reason that the 
loss of miR-145 led to upregulation of membrane-bound ACE in VSMCs, causing chronic stimulation of VSMCs by angiotensin II, which results in desensitization and "angiotensin resistance" of VSMCs $(32,33)$. Desensitization toward angiotensin signaling was also indicated at the molecular level by downregulation of the angiotensin receptor, RGS4, and RGS5 (a regulator of G protein signaling molecules); and changes in Rho signaling and calcium handling, as well as in molecules directly involved in initiation of contraction. These findings corresponded well with the proposed role of RGS4 and RGS5, which interact with the G protein-coupled angiotensin receptor type 1 (34), in receptor desensitization together with RGS2 (35). The downregulation of RGS2 and RGS4 might also account for the impaired response of miR-143/145 vessels to phenylephrine, since $G$ protein-coupled receptor-interacting proteins (GIPs) including RGS2 are important components of the adrenergic receptor signalosome (36). We believe that the downregulation of the above-mentioned key modulators of SMC contraction is a secondary event, since we did not find evidence for a direct regulation of these molecules by miR-143/145, although we cannot completely rule out this possibility.

Agonist-mediated contraction of the vasculature is essential for the control of blood pressure. Hence, mice with an impaired ability to react to agonist-mediated contraction will show aberrant blood pressure regulation. The compromised increase in blood pressure of Mir143/145-KO to angiotensin II infusion matches this expectation and reflects the finding that the contraction of isolated artery explants is impaired in response to acute changes in angiotensin II concentration. The exaggerated decrease in blood pressure in mutant mice after acute administration of the ACE inhibitor captopril argues along the same lines. In this case, the chronic stimulation of SMCs, which is due to the increased local presence of angiotensin II, was disrupted by the inhibitor and resulted in enhanced relaxation of SMCs. In contrast to increased local angiotensin II signaling, serum concentration of angiotensin II was not increased, which is in line with the decreased blood pressure in mutant mice. It must be borne in mind, however, that the angiotensin resistance of $\mathrm{KO}$ mice, i.e., their inability to respond normally to angiotensin II signaling, does not necessarily mean that the decrease in systemic blood pressure is caused solely by improper angiotensin II signaling. This is also illustrated by a previous report describing relatively normal blood pressure in mice that overexpress ACE, although this model, which relies on the duplication of the Ace gene, differs significantly from Mir143/145 mutant mice, which show an upregulation of ACE specifically in SMCs (37).

The impaired contractile response of Mir143/145-KO vessels seems to be due to multiple different yet related mechanisms, which include at least: (a) accumulation of synthetic VSMCs, which do not develop the same contractile force as contractile VSMCs; and (b) specific defects in receptor-mediated signaling. Such mechanisms might - at least in part - be caused by the local increase in ACE in VSMCs, which favors acquisition of a synthetic phenotype (31) and causes desensitization of $G$ protein-coupled signaling.

However, ACE inhibition or blocking of $\mathrm{AT}_{1}$ receptor signaling increased neither the basic contractile ability nor the accumulation of contractile VSMCs, although chronic inhibition of the RAAS by captopril or losartan for 20 days in mutant mice led to the normalization of 248 or 220 of 507 deregulated transcripts, respectively, and to an improvement in receptor-mediated contractility of isolated arteries, which clearly indicates the importance of the miR-145 target ACE for the phenotype of mutant mice. It has been emphasized recently that "authentic" miRNA targets should be sufficiently sensitive to modest reductions in protein production to benefit from miRNA regulation (38). Clearly, ACE fulfills this important new criterion of a physiological significant miRNA target, since ACE activity in VSMCs needs to be tightly controlled to allow stringent regulation by the RAAS. Taken together, our findings strongly suggest a role of increased ACE expression for blood pressure dysregulation in mutant mice but also indicate that ACE is not responsible for all changes observed. Given the general assumption that miRNAs exert their effects by simultaneous regulation of numerous targets, this observation is not surprising and excludes a simple linear model that relies on monocausal relations. The identification of other putative targets that were upregulated in Mir143/145-KO mice further support the idea of miR-143/145 as an integrated signaling node that controls the transition between the synthetic and contractile phenotype of VSMCs. The Mir143/145 gene cluster might also be involved in the separation of smooth muscle properties from other muscle types, since several structural components of SMCs were downregulated, while typical striated muscle molecules were upregulated. This observation fits nicely with the developmental shift of expression of miR-143/145 from cardiac to smooth muscle cells. The combination of targets that act as regulators and effectors of the synthetic and the contractile phenotype is intriguing and argues for a feed-forward mechanism. The integrated control of regulators and downstream targets of such regulators is a major theme in the regulation of cellular differentiation and allows management of complex tasks (39). Our present analysis is based on the combined inactivation of the Mir143 and Mir145 genes, which are located in the same cluster. Although both genes seem to be coexpressed in all cells analyzed, they might not necessarily serve the same function, in particular since the target sequences for the miRNAs differ substantially. Further analysis of single gene knockouts will reveal whether Mir143 and Mir145 genes control distinct processes or whether both genes act together synergistically as a functional unit to regulate smooth muscle phenotype variation.

According to the classic definition, arteriosclerosis is characterized by SMC hyperplasia or hypertrophy and matrix protein accumulation in the intima and/or media with or without lipid deposition, resulting in thickening and stiffness of the arterial wall (40). Arteriosclerosis includes (spontaneous) atherosclerosis, accelerated arteriosclerosis, restenosis after percutaneous transluminal coronary angioplasty, and vein graft atherosclerosis (41). Although the importance of the role of VSMCs in the pathogenesis of atherosclerosis is well established (42), it has not been possible so far to separate effects caused by increased proliferation and secretory activity of VSMCs from other physiological changes, such as hyperlipidemia and chronic inflammation. Our finding that miR-143/145 mutant mice develop neointimal lesions in the absence of hyperlidemia, lipid depositions, and foam cells highlights the potential role of VSMCs in the pathogenetic process leading to atherosclerosis. It clearly indicates that dysregulation of SMCs due to cellautonomous events resulted in accumulation of synthetic VSMCs, which apparently was sufficient to cause development of arteriosclerotic lesions. The massive enhancement of angiotensin II signaling due to the increased expression of ACE will certainly contribute to this process, since increased levels of angiotensin II have been shown to promote atherosclerotic lesions in apoE-deficient mice (43). Moreover, many studies have indicated that inhibition of ACE can induce regression of atherosclerotic lesions (reviewed in ref. 44). 
Our model presents an explanation for these findings and offers the unique opportunity to study the contribution of SMCs to the multifactorial process that results in formation of arteriosclerotic plaques (25). The identification of the Mir143/145 gene cluster as a major regulator of transitions between different phenotypic conditions of VSMCs opens new avenues to manipulate vascular repair and influence the pathogenesis of atherosclerosis.

\section{Methods}

Mice. A 129S7AB2.2 genomic BAC clone (bMQ-446B20; Sanger) was used to generate the targeting vector. Recombination in SW102 cell was employed to replace the miR-143-to-miR-145 genomic region with a galK cassette (45) that was amplified by the primers flanking pre-miR-143 to pre-miR-145 (5'-CACTCCTCCTGCCCAAGAAGAAAGCCGCGCGGCCTGCGTGCGGAGCGCCTGTCTCCCAGCGGCGCGCCTCAGCACTGTCCTGCTCCTT-3'; 5'-TACTCCCCGGAAGGAAATGCCAGGGTGGGAGGGAGACAGATCCAGCTGCTAAGCCATGACGGCGCGCCTGTTGACAATTAATCATCGGCA-3'). The genomic sequence was inserted via gap repair into a pKO targeting vector containing a DTA expression cassette and 450-bp homology arms for BAC recombination, resulting in a vector containing $6.5 \mathrm{~kb}$ of sequence $5^{\prime}$ of miR-143 and $5.5 \mathrm{~kb} \mathrm{3} 3^{\prime}$ of miR-145 from genomic sequence $5^{\prime}$-TGAGCTGCTGGAGGCAAGGCTTGG-3' to $5^{\prime}$ AGCCCAGCCTGGTCTATAGAGGGAG- ${ }^{\prime}$. The AscI restriction sites flanking the galK cassette were used to replace the galK with a floxed IRES-lacZ-Neo ${ }^{\mathrm{R}}$ cassette. The vector was electroporated into MPI II ES cells (46), and recombinant clones were identified. Recombinant ES cells were injected into blastocysts and transferred to pseudopregnant mice. Chimeric animals were backcrossed to C57BL/6 mice. Heterozygous animals were used to obtain WT and KO littermates. ES cells and mice were genotyped using an EcoRV restriction digest and a 5 ' outside probe amplified from genomic DNA using oligonucleotides (5'-GACGGTCCTGAACACCTGGATCCC-3'; 5'-TGCTGTGAGCTCTGAGAACCTCGG-3'). For chronic $\mathrm{ACE}$ or $\mathrm{AT}_{1}$ inhibition, drinking water of mice was supplemented with $600 \mu \mathrm{g} / \mathrm{ml}$ captopril (catalog C-8856; Sigma-Aldrich) and $100 \mu \mathrm{g} / \mathrm{ml}$ losartan (catalog 61188; Fluka) for at least 3 weeks. The experiments were performed in accordance with German legislation on protection of animals and were approved by the local governmental animal care committee (Regierungspraesidium Darmstadt).

Morphological analysis. Arteries were perfused using PBS solution via the heart apex at $100 \mathrm{mmHg}$ pressure for 2 minutes, followed by perfusion with $4 \%$ paraformaldehyde in PBS. After the dissection of different arteries, these were additionally fixed in $3 \%$ glutaraldehyde for another 12 hours at $40^{\circ} \mathrm{C}$ and embedded in Epon. Ultrathin sections were stained with uranyl acetate and lead citrate, viewed, and photographically recorded using a Philips CM 10 electron microscope.

The phenotype of VSMCs was determined ultrastructurally according to previously reported criteria (47). Morphological assessments were done by 2 different researchers unaware of the identity of analyzed samples, In brief, contractile VSMCs are characterized by highly compacted contractile filaments and numerous intracellular electron dense puncta (arrowheads, Figure 3A), which strengthen and support the contractile apparatus, and prominent focal adhesions (arrows, Figure 3A). In contrast, synthetic VSMCs are smaller in size and rich in ER and ribosomes compared with contractile VSMCs and exhibit loosely packed contractile filaments, almost indiscernible focal adhesions, and intracellular electron dense puncta (Figure 3B). The mixed phenotype of VSMCs was defined by focal adhesions located at the cell periphery and scarceness or absence of intracellular electron dense puncta. In addition, ER structures in mixed VSMCs are located only in the perinuclear region, while they are extreme- ly abundant and extend up to cell periphery in synthetic VSMCs. These features clearly distinguish contractile VSMCs from mixed VSMCs. The phenotype of VSMCs in each mouse was quantified in at least 300 cells per 3 arterial segments (minimum $100 \mu \mathrm{m}$ ) using consecutive transversal sections. For detection of $\beta$-galactosidase activities, tissues or embryos were fixed in PBS containing $1 \%$ formaldehyde, $0.2 \%$ glutaraldehyde, $0.2 \%$ NP-40, and $0.1 \%$ sodium deoxycholate for 15-60 minutes, depending on size. Samples were processed as whole tissue or as cryosections. Samples were rinsed in PBS for 15 minutes and stained in PBS containing $2 \mathrm{mM}$ $\mathrm{MgCl}_{2}, 5 \mathrm{mM} \mathrm{K}_{3} \mathrm{Fe}(\mathrm{CN})_{6}, 5 \mathrm{mM} \mathrm{K}_{4} \mathrm{Fe}(\mathrm{CN})_{6}, 1 \mathrm{mg} / \mathrm{ml} \mathrm{X}$-gal, and $0.2 \% \mathrm{NP}-40$ and $0.1 \%$ sodium deoxycholate. For embryos younger than $\mathrm{E} 12.5$, solutions contained $0.02 \%$ NP-40 and no sodium deoxycholate.

Western blotting and immunofluorescence. Protein lysates $(10 \mu \mathrm{g})$ were separated on 4\%-12\% SDS-PAGE gradient gels and transferred onto nitrocellulose membranes (Invitrogen). Immunoreactive proteins were visualized with corresponding HRP-conjugated secondary antibodies using SuperSignal West Femto detection solutions (Thermo Scientific). Signals were detected and analyzed with a Bio-Rad Versadoc System and Quantity One. We used polyclonal antisera against ACE-1 (catalog ab39172; Abcam), NCS-1 (catalog sc-13037; Santa Cruz Biotechnology Inc.), and collagen type I (catalog C-2456; Sigma-Aldrich) and monoclonal antibodies against $\alpha$-SMA (catalog C-6198; Sigma-Aldrich), F4/80 (catalog MF48004; Invitrogen), caveolin-3 (catalog 610420; BD), caldesmon-1 (catalog C-4562, clone hHCD; Sigma-Aldrich), GAPDH (catalog 2118; NEB), and calponin (catalog C2687, clone hCP; Sigma-Aldrich). Secondary antibodies were from Pierce, Thermo Scientific (goat anti-rabbit HRP, catalog 1858415; goat anti mouse HRP, catalog 1858413) or Invitrogen (Alexa Fluor).

Microarrays and Northern blot analysis. Tissues were dissected from PBS-perfused animals, and total RNA was isolated using the TRIzoL method (Invitrogen). RNA quality was checked on the Agilent 2100 Bioanalyzer using the RNA 6000 Nano Kit. For mRNA expression analysis, the Affymetrix GeneChip Mouse Genome 430 2.0 Array was employed with the respective 1-cycle target labeling protocol. Data were analyzed by the RMA algorithm using the Affymetrix Expression Console. Data are available at http://www. mpi-bn.mpg.de/en/research/heart/boettger/downloads.html. An unpaired $t$ test was performed with $\log _{2}$-transformed data to identify significantly differentially expressed transcripts, and FC was calculated using DNAStar ArrayStar 3.0 software. Among the probe sets, 507 were identified as having $\mathrm{FC}>1.5$ or $\mathrm{FC}<0.666(P<0.05)$.

For miRNA expression analysis, 384 3' C6 amino linker DNA oligonucleotides complementary to published miRNA sequences (miRBase; http:// microrna.sanger.ac.uk) were synthesized and spotted with 8 replicates per array to Nexterion E slides. Probe labeling and hybridization followed the Agilent miRNA labelling protocol, but P-CTTTT-Alexa555 or P-CTTTTAlexa647 oligonucleotides (MWG-Biotech) were ligated to the miRNAs. Arrays were scanned and analyzed with an Axon 4200B scanner and GenePix Pro 6.0. Normalization and data analysis was performed using Acuity 4.0 (Molecular Devices). For multiple tissue comparison, miRNA from tissues was labeled with Alexa Fluor 555 dye; miRNA from whole E15.5 embryos labeled with Alexa Fluor 647 dye was used as a reference. Data were linear normalized to a log ratio of 1 for the ratio of medians of let-7a-g and let-7i signals. The $\log _{2}$ ratio data were centered for the single miRNA expression signals (by subtracting the row mean from each value); $\log _{2}$ ratio data are shown. For Northern blot analysis, $3 \mu \mathrm{g}$ of total RNA (with the exception of the aorta, for which $1.5 \mu \mathrm{g}$ was used) was separated on a $15 \%$ polyacrylamide-urea gel (Invitrogen) and blotted on Hybond XL (Amersham). miR-145, miR-143, and U6 antisense oligonucleotides were labeled with $\gamma$-ATP and PNK (NEB) and purified using Micro Bio-Spin 6 columns (Bio-Rad). Hybridization was performed in ULTRAhyb hybridiza- 
tion buffer (Ambion) at $30^{\circ} \mathrm{C}$ overnight. Blots were washed 2 times in $2 \times$ SSC $/ 0.1 \%$ SDS at $25^{\circ} \mathrm{C}(5$ minutes $)$ and at $42^{\circ} \mathrm{C}(15$ minutes $)$. Signals were detected using a BAS-2500 imager (Fujifilm).

Quantitative proteomics. Aortae were dissected from ${ }^{13} \mathrm{C}_{6}$ Lys-labeled "heavy" WT mice and from nonlabeled WT and KO littermates. Protein extracts were processed and mass spectroscopy was performed as described previously (15). Briefly, all LC-MS/MS measurements were performed with an LTQ-Orbitrap (Thermo Scientific) combined with an Agilent 1200 nanoflow HPLC system. The mass spectrometer was operated in the datadependent mode to automatically measure full MS scans and MS/MS spectra. Peptides were identified by searching against the International Protein Index sequence database (mouse IPI, version 3.24; http://www.ebi.ac.uk/ IPI/IPIhelp.html) using the Mascot search algorithm (http://www.matrixscience.com). Mass spectra were analyzed by the MaxQuant software package (http://www.maxquant.org; refs. 48, 49), which performs peak lists, SILAC quantification, and false positive rate determination (50). Ratios and $P$ values were calculated from the mean of $3{ }^{13} \mathrm{C}_{6}$ Lys-WT/WT and 3 ${ }^{13} \mathrm{C}_{6}$ Lys-WT/KO ratios.

Identification of miRNA targets. miRNA target predictions were downloaded from miRBase (http://microrna.sanger.ac.uk; Mus musculus Targets Release Version 5, release 2008-01-08, miRanda-based), from microrna. org (http://www.microrna.org/; mouse miRNA Target Site predictions release 2008-01-08; miRanda-based) (17), and from TargetScan.org (http://www.targetscan.org; version 4.2, predicted targets of conserved families) (19). Lists of mRNAs (via Affymetrix ID) or proteins (via gene symbol) that were significantly regulated in WT versus KO mice, but not affected by chronic captopril treatment, were compared with lists of targets predicted for miR-143 and miR-145 using the MatchMiner database (http://discover.nci.nih.gov/matchminer/) (20).

Hemodynamic measurements. Aortic catheterization was performed using a $1.4 \mathrm{~F}$ pressure-volume catheter. Data were recorded and analyzed with Chart version 5.4 (ADInstruments) as described previously (51). Mice were anesthetized with isoflurane $\left(2 \% \mathrm{vol} / \mathrm{vol}\right.$ in $\left.\mathrm{O}_{2}\right)$, and their body temperature was kept at $37^{\circ} \mathrm{C}$ as monitored by rectal probe. Captopril $(30 \mathrm{mg} / \mathrm{kg}$ body weight, $50 \mu \mathrm{l}$ ) and angiotensin II ( 5 to $50,000 \mathrm{ng} / \mathrm{kg}$ body weight, $50 \mu \mathrm{l})$ were applied via the left jugular vein.

For measurements of conscious, unrestrained mice, hemodynamic parameters were recorded by telemetry (DSI, Transoma Medical; TA11PAC10 transmitters) after a recovery period from surgery of more than 7 days. Recordings were obtained every 2 minutes for 20 seconds at a sampling frequency of $1,000 \mathrm{~Hz}$ and were analyzed for day- (0700-1900 hours) and nighttime (1900-0700 hours) periods, respectively, to evaluate circadian variation in hemodynamics.

Myograph experiments. Isolated segments of femoral and mesenteric arteries were examined in an isometric microvessel myograph as described previously (52). Mounted vessels were stimulated with $80 \mathrm{mM} \mathrm{K}+30 \mathrm{nM}$ angiotensin II, or increasing concentrations of phenylephrine (100 nM to $10 \mu \mathrm{M})$. The $\mathrm{pCa}$-force relationships $(13,14)$ were obtained in permea- bilized mouse femoral artery segments. In brief, arteries were permeabilized for 30 minutes using $0.5 \%$ Triton $\mathrm{X}-100$ in relaxation solution. The latter consisted of (in mM): EGTA 1, Pipes 30, $\mathrm{Na}_{2} \mathrm{ATP} 5.16, \mathrm{MgCl}_{2} 7.31$, sodium creatine phosphate 10 , and potassium gluconate 74.1 . For contraction curves, the buffer was supplemented with $160 \mathrm{U} / \mathrm{ml}$ calmodulin and $100 \mathrm{U} / \mathrm{ml}$ creatine phosphokinase. The concentration of free $\mathrm{Ca}^{2+}$ ions was calculated as described previously (53).

Analysis of blood gas and gut transit time. Blood was collected after mice were sacrificed by decapitation and was analyzed using an i-Stat 1 Analyzer with EG6+ cartridges (Abbott Laboratories). For analysis of gut transit time, after a 20-hour fasting period, mice were force fed $0.3 \mathrm{ml}$ $2.5 \%$ Evans Blue (catalog E-2129; Sigma-Aldrich) in 1.5\% methylcellulose (catalog E-2129; Fluka). Mice were returned to cages with water and food, and time until appearance of stained feces was recorded.

Analysis of blood circulating angiotensin II and aortic cGMP concentration. Blood serum samples were obtained by cardiac puncture using Bestatin angiotensinase inhibitor solution (Bühlmann GmbH). Angiotensin II concentrations were determined using an angiotensin II RIA after reverse phase extraction of plasma samples using columns provided with the RIA (Bühlmann GmbH). To match the volume requirement of the RIA, a part of the plasma samples analyzed ( $n=5 \mathrm{WT}, n=4 \mathrm{KO}$ ) was obtained by pooling plasma of a maximum of 2 animals of the same genotype. Aortae from WT and KO mice were snap-frozen in liquid nitrogen and homogenized in 5\% TCA. cGMP concentration was determined with a cGMP EIA Kit (Cayman Chemicals) using the acetylated cGMP protocol.

Statistics. Data are presented as mean \pm SEM and were tested by $t$ test or 1- or 2-way ANOVA. $P$ values less than 0.05 were considered statistically significant.

\section{Acknowledgments}

The authors thank Angela Bachmann for collaboration with miRNA arrays and Ulrike Neckmann, Ulrike Schlapp, Katja Kolditz, and Susanne Kreuzer for technical help. We are grateful to J. Cox and M. Mann for giving us access to the MaxQuant software package. This work was supported by the Max-Planck-Society, the DFG (Br1416), the EU Commission (MYORES Network of Excellence), the Kerckhoff-Foundation, and the Excellence Initiative "Cardiopulmonary System" (in support of T. Braun) and "Bioss" EXC294 (in support of L. Hein and N. Beetz).

Received for publication February 9, 2009, and accepted in revised form July 8, 2009.

Address correspondence to: Thomas Braun or Thomas Boettger, Max-Planck-Institut für Herz- und Lungenforschung, Parkstrasse 1, D-61231 Bad Nauheim, Germany. Phone: 49-6032-705-401; Fax: 49-6032-705-419; E-mail: thomas.braun@kerckhoff.mpg.de (T. Braun); thomas.boettger@mpi-bn.mpg.de (T. Boettger).
1. Owens, G.K., Kumar, M.S., and Wamhoff, B.R. 2004. Molecular regulation of vascular smooth muscle cell differentiation in development and disease. Physiol. Rev. 84:767-801.

2. Rensen, S.S., Doevendans, P.A., and van Eys, G.J. 2007. Regulation and characteristics of vascular smooth muscle cell phenotypic diversity. Neth. Heart J. 15:100-108.

3. Doran, A.C., Meller, N., and McNamara, C.A. 2008. Role of smooth muscle cells in the initiation and early progression of atherosclerosis. Arterioscler. Thromb. Vasc. Biol. 28:812-819.

4. Ross, R. 1995. Cell biology of atherosclerosis. Annu. Rev. Physiol. 57:791-804.
5. Bartel, D.P. 2004. MicroRNAs: genomics, biogenesis, mechanism, and function. Cell. 116:281-297.

6. Landgraf, P., et al. 2007. A mammalian microRNA expression atlas based on small RNA library sequencing. Cell. 129:1401-1414.

7. Zhao, Y., et al. 2007. Dysregulation of cardiogenesis, cardiac conduction, and cell cycle in mice lacking miRNA-1-2. Cell. 129:303-317.

8. Makeyev, E.V., Zhang, J., Carrasco, M.A., and Maniatis, T. 2007. The microRNA miR-124 promotes neuronal differentiation by triggering brain-specific alternative pre-mRNA splicing. Mol. Cell. 27:435-448.

9. Leucht, C., et al. 2008. MicroRNA-9 directs late organizer activity of the midbrain-hindbrain boundary. Nat. Neurosci. 11:641-648.

10. Ruzicka, D.L., and Schwartz, R.J. 1988. Sequential activation of alpha-actin genes during avian cardiogenesis: vascular smooth muscle alpha-actin gene transcripts mark the onset of cardiomyocyte differentiation. J. Cell Biol. 107:2575-2586.

11. Li, L., Miano, J.M., Cserjesi, P., and Olson, E.N. 1996. SM22 alpha, a marker of adult smooth muscle, is expressed in multiple myogenic lineages during embryogenesis. Circ. Res. 78:188-195.

12. Rishniw, M., Xin, H.B., Deng, K.Y., and Kotlikoff, M.I. 2003. Skeletal myogenesis in the mouse esophagus does not occur through transdifferentiation. 
Genesis. 36:81-82.

13. Pfitzer, G., and Boels, P.J. 1991. Differential skinning of smooth muscle: a new approach to excitationcontraction coupling. Blood Vessels. 28:262-267.

14. Khromov, A.S., et al. 2006. Smooth muscle of telokin-deficient mice exhibits increased sensitivity to $\mathrm{Ca} 2+$ and decreased cGMP-induced relaxation. Proc. Natl. Acad. Sci. U. S. A. 103:2440-2445.

15. Kruger, M., et al. 2008. SILAC mouse for quantitative proteomics uncovers kindlin-3 as an essential factor for red blood cell function. Cell. 134:353-364.

16. John, B., et al. 2004. Human microRNA targets. PLoS Biol. 2:e363.

17. Betel, D., Wilson, M., Gabow, A., Marks, D.S., and Sander, C. 2008. The microRNA.org resource: targets and expression. Nucleic Acids Res. 36:D149-D153.

18. Griffiths-Jones, S., Saini, H.K., van Dongen, S., and Enright, A.J. 2008. miRBase: tools for microRNA genomics. Nucleic Acids Res. 36:D154-D158.

19. Grimson, A., et al. 2007. MicroRNA targeting specificity in mammals: determinants beyond seed pairing. Mol. Cell. 27:91-105.

20. Bussey, K.J., et al. 2003. MatchMiner: a tool for batch navigation among gene and gene product identifiers. Genome Biol. 4:R27.

21. Abouhamed, M., Reichenberg, S., Robenek, H., and Plenz, G. 2003. Tropomyosin 4 expression is enhanced in dedifferentiating smooth muscle cells in vitro and during atherogenesis. Eur. J. Cell Biol. 82:473-482

22. Potapova, I.A., Cohen, I.S., and Doronin, S.V. 2007. Voltage-gated ion channel Kv4.3 is associated with Rap guanine nucleotide exchange factors and regulates angiotensin receptor type 1 signaling to small G-protein Rap. FEBS J. 274:4375-4384.

23. Miralles, F., Posern, G., Zaromytidou, A.I., and Treisman, R. 2003. Actin dynamics control SRF activity by regulation of its coactivator MAL. Cell. 113:329-342.

24. Ji, R., et al. 2007. MicroRNA expression signature and antisense-mediated depletion reveal an essential role of microRNA in vascular neointimal lesion formation. Circ. Res. 100:1579-1588.

25. Xu, Q. 2004. Mouse models of arteriosclerosis: from arterial injuries to vascular grafts. Am. J. Pathol. 165:1-10.

26. Farh, K.K., et al. 2005. The widespread impact of mammalian microRNAs on mRNA repression and evolution. Science. 310:1817-1821.

27. Wang, D., et al. 2001. Activation of cardiac gene expression by myocardin, a transcriptional cofactor for serum response factor. Cell. 105:851-862.

28. Xu, N., Papagiannakopoulos, T., Pan, G., Thomson, J.A., and Kosik, K.S. 2009. MicroRNA-145 regulates OCT4, SOX2, and KLF4 and represses pluripotency in human embryonic stem cells. Cell. 137:647-658.

29. Dzau, V.J. 2001. Theodore Cooper Lecture: Tissue angiotensin and pathobiology of vascular disease: a unifying hypothesis. Hypertension. 37:1047-1052.

30. Touyz, R.M. 2005. Intracellular mechanisms involved in vascular remodelling of resistance arteries in hypertension: role of angiotensin II. Exp. Physiol. 90:449-455.

31. Schieffer, B., et al. 2000. Expression of angiotensin II and interleukin 6 in human coronary atherosclerotic plaques: potential implications for inflammation and plaque instability. Circulation. 101:1372-1378.

32. Griendling, K.K., et al. 1987. Correlation of receptor sequestration with sustained diacylglycerol accumulation in angiotensin II-stimulated cultured vascular smooth muscle cells. J. Biol. Chem. 262:14555-14562.

33. Lassegue, B., et al. 1995. Angiotensin II down-regulates the vascular smooth muscle AT1 receptor by transcriptional and post-transcriptional mechanisms: evidence for homologous and heterologous regulation. Mol. Pharmacol. 48:601-609.

34. Wang, X., et al. 2008. RGS5, RGS4, and RGS2 expression and aortic contractibility are dynamically co-regulated during aortic banding-induced hypertrophy. J. Mol. Cell. Cardiol. 44:539-550.

35. Hercule, H.C., et al. 2007. Regulator of $G$ protein signalling 2 ameliorates angiotensin II-induced hypertension in mice. Exp. Physiol. 92:1014-1022.

36. Lyssand, J.S., et al. 2008. Blood pressure is regulated by an alpha1D-adrenergic receptor/dystrophin signalosome. J. Biol. Chem. 283:18792-18800.

37. Krege, J.H., et al. 1997. Angiotensin-converting enzyme gene mutations, blood pressures, and cardiovascular homeostasis. Hypertension. 29:150-157.

38. Seitz, H. 2009. Redefining microRNA targets. Curr. Biol. 19:870-873.

39. Penn, B.H., Bergstrom, D.A., Dilworth, F.J., Bengal, E., and Tapscott, S.J. 2004. A MyoD-generated feedforward circuit temporally patterns gene expression during skeletal muscle differentiation. Genes Dev. 18:2348-2353.

40. Stary, H.C., et al. 1994. A definition of initial, fatty streak, and intermediate lesions of atherosclerosis. A report from the Committee on Vascular Lesions of the Council on Arteriosclerosis, American Heart Association. Circulation. 89:2462-2478.

41. Ip, J.H., et al. 1990. Syndromes of accelerated atherosclerosis: role of vascular injury and smooth muscle cell proliferation. J. Am. Coll. Cardiol. 15:1667-1687.

42. Ross, R., and Glomset, J.A. 1973. Atherosclerosis and the arterial smooth muscle cell: proliferation of smooth muscle is a key event in the genesis of the lesions of atherosclerosis. Science. 180:1332-1339.

43. Daugherty, A., Manning, M.W., and Cassis, L.A. 2000. Angiotensin II promotes atherosclerotic lesions and aneurysms in apolipoprotein E-deficient mice. J. Clin. Invest. 105:1605-1612.

44. Halkin, A., and Keren, G. 2002. Potential indications for angiotensin-converting enzyme inhibitors in atherosclerotic vascular disease. Am. J. Med. 112:126-134.

45. Warming, S., Costantino, N., Court, D.L., Jenkins, N.A., and Copeland, N.G. 2005. Simple and highly efficient BAC recombineering using galK selection. Nucleic Acids Res. 33:e36.

46. Voss, A.K., Thomas, T., and Gruss, P. 1997. Germ line chimeras from female ES cells. Exp. Cell Res. 230:45-49.

47. Scholz, D., et al. 2000. Ultrastructure and molecular histology of rabbit hind-limb collateral artery growth (arteriogenesis). Virchows Arch. 436:257-270.

48. Cox, J., and Mann, M. 2007. Is proteomics the new genomics? Cell. 130:395-398.

49. Graumann, J., et al. 2008. Stable isotope labeling by amino acids in cell culture (SILAC) and proteome quantitation of mouse embryonic stem cells to a depth of 5,111 proteins. Mol. Cell. Proteomics. 7:672-683.

50. Kersey, P.J., et al. 2004. The International Protein Index: an integrated database for proteomics experiments. Proteomics. 4:1985-1988.

51. Brede, M., et al. 2002. Feedback inhibition of catecholamine release by two different alpha2-adrenoceptor subtypes prevents progression of heart failure. Circulation. 106:2491-2496

52. Brede, M., et al. 2001. Vascular hypertrophy and increased P70S6 kinase in mice lacking the angiotensin II AT(2) receptor. Circulation. 104:2602-2607.

53. Fabiato, A., and Fabiato, F. 1979. Calculator programs for computing the composition of the solutions containing multiple metals and ligands used for experiments in skinned muscle cells. J. Physiol. (Paris). 75:463-505. 